\title{
Do jumps help in forecasting the density of returns? *
}

\author{
Julien Chevallier ${ }^{\dagger}$ \\ Université Paris Dauphine
}

\author{
Florian Ielpo \\ Lombard Odier Investment Managers \\ Université Paris 1
}

\author{
Benoît Sévi ${ }^{\S}$ \\ Aix-Marseille School of Economics
}

First version: December 2009

This version: July 2011

\begin{abstract}
The estimation of the jump component in asset pricing has witnessed a considerably growing body of literature. Of particular interest is the decomposition of total volatility between its continuous and jump components. Recent contributions highlight the importance of the jump component in forecasting the volatility at different horizons. In this paper, we extend the methodology developed by Maheu and McCurdy (2011) to measure the information content of intraday data in forecasting the density of returns at horizons up to 60 days. We extract jumps as in Andersen, Bollerslev, Frederiksen and Nielsen (2010) to have a measure of the jumps in returns. Then, we estimate a bivariate model of returns and volatilities where the jump component is independently modeled. Our empirical results for S\&P 500 futures, WTI crude oil futures, and the USD/JPY exchange rate confirm the importance of considering the continuous/jump decomposition for density forecasting.
\end{abstract}

JEL Classification: C15, C32, C53, G1

Keywords: density forecasting, jumps, realized volatility, bipower variation, median realized volatility, bivariate model.

\footnotetext{
* Part of the paper was written while Julien Chevallier and Benoît Sévi were visiting researchers at, respectively, the Imperial College London Business School and the London Business School. The authors are thankful to Walter Distaso, Per Frederiksen, Rehim Kilic, Robert Kosowski, John Maheu, Nour Meddahi, Diaa Noureldin, Petra Posedel, Anders Rahbek, Kevin Sheppard, Stephen Taylor, Almut Veraart and Filip Zikes for many insightful comments. Helpful comments were received from participants at the 2009 Université Paris Ouest Econometrics Workshop, the 2nd Humboldt-Copenhagen Conference on Financial Econometrics and the 18th International Conference on Forecasting Financial Markets in Marseille.

${ }^{\dagger}$ Corresponding Author. CGEMP/LEDa, Place du Maréchal de Lattre de Tassigny, 75775 Paris Cedex 16, France. Tel: +33(0)144054464. Fax: +33(0)144054484. julien.chevallier@dauphine.fr

${ }^{\ddagger}$ Lombard Odier Asset Management, rue de la Corraterie 11, 1204 Geneva, Switzerland and University Paris 1 Panthéon - CERMSEM, Maison des Sciences Economiques, 106 Boulevard de l'Hôpital, 75013 Paris, France. Email: florian.ielpo@ensae.org

$\S^{\S}$ DEFI, Château La Farge, Route des Milles, 13290 Aix-en-Provence Les Milles, France. Email: benoit.sevi@gmail.com
} 


\section{Introduction}

When it comes to forecasting the distribution of returns for risk management purposes, is the separation between volatility and jumps equally important? In a reference paper, Andersen et al. (2007) provide empirical evidence that disentangling jumps from the continuous component significantly help in forecasting the realized volatility at horizons up to 22 days. The explanation for this result is the strong persistence in the continuous component and the absence of autocorrelation in the jump component. Recently, Hansen et al. (2011), Shephard and Sheppard (2010), and Maheu and McCurdy (2011) have suggested "complete" models of returns and volatility. In particular, Maheu and McCurdy (2011) propose a bivariate specification of returns and volatility to obtain density forecasts at horizons up to 60 days. They confirm, in the density context, numerous previous findings that intraday data improve forecasts. The estimation of a multivariate models possesses interesting characteristics compared to the separate estimation of an univariate model. Namely, multivariate models allow to obtain densities forecasts. ${ }^{1}$ Maheu and McCurdy (2011) allow for a rich underlying distribution in the return equation, by using mixture of normals (see Bertholon et al., 2006). We merge these two strands of this recent literature to investigate whether the separation between the continuous and the jump components is of central importance in predicting the density of returns. Our results strongly argue in favor of separating the two components when forecasting the density of returns up to 60 days. Indeed, disentangling jumps from the continuous component help in forecasting the density of returns.

Econometric methods used to disentangle jumps and volatility are unveiling new empirical questions. While recent developments in financial econometrics allow to derive better forecasts of return densities (see Corradi and Swanson (2006) for a recent survey), the issue of the inclusion of the jump component and its information content for such a purpose has not been investigated to date. Hence, in this paper, we examine whether this refinement to use a clear differentiation between jumps and continuous volatility is empirically worth the trouble in a density forecasting exercise. We conduct such an analysis at various horizons (up to 60 days), thus allowing a very detailed analysis of the effects we are interested in.

Forecasting density is essential in empirical finance applications such as portfolio choice, risk management activities or derivatives pricing. Each activity requires indeed a full specification of the return distribution. Forecasts of the future values of economic variables are used widely in decision making. Point forecasts, the often traditional focus, are better seen as the central points of ranges of uncertainty. Consequently, to provide a complete description of the uncertainty associated with the point forecast many professional forecasters now publish density forecasts, or more popularly fan charts. In contrast to interval forecasts, which give the probability that the outcome will fall within a stated interval, density forecasts provide a complete description of the uncertainty associated with a forecast. They can thus be seen to provide information on

\footnotetext{
${ }^{1}$ It has also advantages when residuals of univariate models are contemporaneously correlated as highlighted in Bollerslev et al. (2009). We do not consider here the possible correlation between errors in single equations, which is left for further research.
} 
all possible intervals. In conjunction with the increased use of density forecasts by professional forecasters and central banks, the academic literature has also devoted increased emphasis to density forecasting (for surveying methods for predictive density evaluation, see among others Tay and Wallis (2000), Clements (2005), Timmermann (2006) and Wallis (2007)).

This paper adopts the parsimonious specification of the Heterogeneous Autoregressive Model of the Realized Volatility (HAR-RV) model by Corsi (2009) to capture the well-known longmemory dependence in volatility. We also proceed with the detection of jumps following Huang and Tauchen's (2005) statistical test relying on the bipower variation (BPV) estimator. We adapt the test statistic to the newly developed median realized volatility (MedRV) following the empirical work by Theodossiou and Zikes (2009) showing the interesting properties of this estimator.

By using intraday data, it is possible to extract jumps as the difference, when statistically significant, between realized volatility and bipower variation/median realized volatility. This decomposition allows to include (or not) jumps for forecasting purposes. Based on the link between the conditional variance and the realized volatility highlighted by Maheu and McCurdy (2011), we estimate two kinds of models: (i) EGARCH models based on daily data, and (ii) bivariate models based on intraday data including jumps (realized volatility) or not (bipower variation, median realized volatility). The motivation behind considering jump-robust measures for realized volatility is that they simply have better predictive properties than non-jump-robust ones (see Shephard and Sheppard (2011), footnote 3).

As mentioned above, we rely on Maheu and McCurdy's (2011) bivariate model to simultaneously estimate returns and volatilities, while assuming a possible leverage effect. The cornerstone of the model is the link that the authors establish between some realized volatility estimators and the "true" conditional volatility in light of the theory underlying these estimators. Such a model allows to derive density forecasts from intraday data which can be compared between them or to standard EGARCH models. This comparison between EGARCH and bivariate specifications for daily returns and realized volatilities ("naive" and jump-robust estimators) is conducted by using the predictive likelihood of returns (Diebold and Mariano (1995) and Amisano and Giacomini (2007)). We find that intraday data convey most of the asset's informational content, as discussed by Taylor and Xu (1997), among others, for the point forecast case. Recall that the central goal of the paper is to assess the contribution of jumps in forecasting the density of returns thereby extending our understanding of the information contained in the different components of the volatility process. The bivariate model is estimated through maximum likelihood with possibly a mixture of normals, which allows to improve significantly the goodnessof-fit of the model.

This paper makes three contributions: 1) we extend the framework of Maheu and McCurdy (2011) and show how to model jumps in their bivariate framework, 2) we confirm their findings that intraday data yield to better densities forecasts than daily data for a larger set of financial series, and 3) we assess the importance of jumps when forecasting the density of returns by 
comparing jump-robust and non-jump-robust measures of realized volatilities. Compared to the "naive" measure of realized volatility, considering jumps specifically provides significant improvement on the accuracy of forecasts of return densities. We thus extend the results in Andersen et al. (2007) (see also Corsi et al. (2010)) in showing the importance of disentangling jumps from the continuous component for forecasting purposes.

The remainder of the paper is organized as follows. Section 2 details our modeling strategy for jumps, the choice of volatility estimators using intraday data and the methodology to estimate the bivariate model. Section 3 discusses the empirical results. Section 4 concludes.

\section{Volatility, jumps and discrete time series model specification}

In this section, we present first the time series models, second the parameter estimation procedures, and third the density forecast comparison tests.

\section{$2.1 \quad$ Time series models}

The aim of our article is to present empirical evidence regarding the interest of disentengling jumps from volatility when it comes to forecasting the density of returns. To do so, we evaluate the relative forecasting performances of different discrete time time series models. These models are selected for their ability to handle non-Gaussian distributions and time varying volatility. Jumps have indeed an effect on both the unconditional distribution of returns and volatility. Their impact on times series models can be of three kinds:

- Jumps can be captured through the conditional distribution chosen in the discrete time series models.

- Jumps can impact the measurement of volatility, as revealed by the now large literature on realized risk measures.

- Jumps can impact the dynamics of volatility, as in most time series models the conditional volatility is computed from past returns or residuals that incorporate a jumps component by essence.

When a model would be able to handle these three aspects in a way that disentangles jumps from volatility components, the question of its superior ability to produce density forecast would still require to be considered. We base our empirical work on the three following time series models:

- A conditionally Gaussian model:

$$
r_{t}=\mu+\sigma_{t} \epsilon_{t},
$$


with $\epsilon_{t} \sim N(0,1)$. This model naturally ignores jumps. The only source of leptokurticity in the returns' process comes from the time varying behavior of volatility.

- A model based on a mixture of two Gaussian distributions for its conditional distribution:

$$
r_{t}=\mu+\sigma_{t} \epsilon_{t},
$$

with $\epsilon_{t} \sim M N\left(\theta, \mu_{1}, \sigma_{1}, \mu_{2}, \sigma_{2}\right)$. This model accomodates jumps in two different ways: first, jumps are captured through $\epsilon_{t}$ that is obtained by mixing two different Gaussian densities. As presented in Bertholon et al. (2006), this distribution is able to span a very large scope of couples of kurtosis and skewness. Possibly, this distribution is consistent with a mixture of a Gaussian distribution and of an extreme-type of jumps (see Section 4.2. in Bertholon et al. (2006)). However for most of the methodologies used here, conditional volatilities $\sigma_{t}$ are functions of $\epsilon_{t-1}$. With this modelling approach, the dynamics of volatility is a function of past jumps.

- A model mixing a conditionally Gaussian distribution with jumps:

$$
r_{t}=\mu+\sigma_{t} \epsilon_{t}+\sum_{i=0}^{N_{t}} x_{i, t},
$$

with $\epsilon_{t} \sim N(0,1), x_{i, t} \sim N\left(\mu_{x}, \sigma_{x}\right)$ and $N_{t} \sim \mathcal{P}(\lambda)$. With such an approach, the past volatility is no longer a function of past jumps, as $\epsilon_{t}$ has been cleansed from the jump component. This latter component is assumed to be captured by $\sum_{i=0}^{N_{t}} x_{i, t}$, that is through a separated component.

For each of these models, the structure for the continuous volatility is an Heterogenous Autoregressive Model, as presented in Corsi (2009). We propose to use this specification jointly with different high frequency measures of volatility:

- The realized variance for day $d$ that is given by the sum of squared intraday returns:

$$
R V_{d, M}=\sum_{j=1}^{M} r_{d, j}^{2}
$$

where the $r_{d, j}$ are intraday returns computed as $r_{d, j}=p_{d, j}-p_{d, j-1}$ for $j=1, \ldots, M . p_{d, j}$ are intraday observations allowing to compute $M$ continuously compounded intraday returns each day.

- Barndorff-Nielsen and Shephard (2004)'s bipower variation (BPV) measure, which is computed as the scaled summation of the product of adjacent absolute returns. Formally, BPV is defined as follows:

$$
B P V_{d, m}=\xi_{1} \sum_{j=1}^{M-1}\left|r_{d, j+1}\right|\left|r_{d, j}\right|
$$


where $\xi_{p} \equiv 2^{p / 2} \Gamma\left(\frac{1 / 2(p+1)}{\Gamma(1 / 2)}\right)=E\left(|Z|_{p}\right)$ denotes the mean of the absolute value of standard normally distributed random variable ${ }^{2}, Z$. The BPV is a consistent estimator of integrated volatility, and allows to decompose the realized volatility into its diffusive and non-diffusive parts. As the sampling frequency increases, the presence of jumps should have no impact because the return representing the jump is multiplied by a non-jump return which tends to zero asymptotically. This is true in case of rare jumps (one each day) when the probability of two consecutive jumps is negligible.

- Nevertheless, the BPV can be upward or downward biased in empirical applications as the sampling frequency is not high enough to eliminate the influence of jumps (or in presence of zero-return). This has motivated the need for alternative estimators which do not suffer from this weakness. Recently, Andersen et al. (2009) suggested the following median realized volatility (MedRV) estimator:

$$
M e d R V_{N}=\frac{\pi}{6-4 \sqrt{3}+\pi}\left(\frac{N}{N-2}\right) \sum_{i=2}^{N-1} \operatorname{med}\left(\left|\Delta Y_{i-1}\right|,\left|\Delta Y_{i}\right|,\left|\Delta Y_{i+1}\right|\right)^{2}
$$

The MedRV estimator has two main advantages: first, the impact of jumps completely vanishes except in the case of two consecutive jumps (which is quite rare at the sampling frequencies used in our empirical application) and second, the estimator is more robust to occurence of zero-returns. ${ }^{3}$

In Section 3, we discuss different empirical key elements related to the use of such volatility measures, such as the intra-day sampling frequency. Using both the BPV and the MedRV, we present in the meantime a way to estimate jumps from intra-day time series. For the time being, we assume that jumps are observable.

Now, we discuss the joint dynamics of volatility and returns. Maheu and McCurdy (2011) relate the conditional variance of daily returns $\sigma_{t}^{2}$ to the realized volatility estimator through a crossequation restriction. Barndorff-Nielsen and Shephard (2002) and Andersen et al. (2003) show that under empirically realistic assumptions, the conditional variance of daily returns should equal the conditional expectation of quadratic variation, or:

$$
E_{t-1}\left(Q V_{t}\right)=\operatorname{Var}_{t-1}\left(r_{t}\right) \equiv \sigma_{t}^{2},
$$

\footnotetext{
${ }^{2}$ This notation is used consistently in the paper.

${ }^{3}$ We could use other estimators to obtain measures of integrated variance, such as QRV (Christensen et al., 2009) estimator, which are shown to be more robust in the presence of microstructure noise and zero-returns. A comparison of these estimators and their properties for density forecasting is beyond the scope of this paper and left for future research.
} 
where $E_{t-1}$ stands for the conditional expectation at time $t-1, Q V_{t}$ the quadratic variation, $\operatorname{Var}_{t-1}\left(r_{t}\right)$ and $\sigma_{t}^{2}$ the conditional variance of returns. Assuming that $R V_{t}$ is an unbiased estimator of $Q V_{t}$, it follows that:

$$
\sigma_{t}^{2}=E_{t-1}\left(R V_{t}\right)
$$

In other words, the one-period-ahead conditional expectation of the realized volatility should equal the "true" conditional volatility assuming the unbiasedness of the realized volatility estimator. Under the assumption of a log-normal distribution for the realized volatility ${ }^{4}$, the conditional expectation can then be expressed as:

$$
\sigma_{t}^{2}=E_{t-1}\left(R M_{t}\right)=\exp \left(E_{t-1} \log \left(R M_{t}\right)+\frac{1}{2} \operatorname{Var}_{t-1}\left(\log \left(R M_{t}\right)\right)\right) .
$$

with $R M_{t}$ a given realized measure $\left(R V_{t}, B P V_{t}, M e d R V_{t}\right)$.

We now turn to the specification of a predictive model for realized volatility. The HAR-RV model initially developed in Corsi (2009) has been used with success in a number of recent contributions (Andersen et al. (2007), Corsi et al. (2008), Liu and Maheu (2009), among others). The economic intuition behind this model is that different groups of investors have different investment horizons, and consequently behave differently (see Muller et al. (1997) for the presentation of the $\mathrm{HARCH}$ original model relying on the Heterogeneous Hypothesis). The genuine HAR-RV model is formally a constrained AR(22) model using RV as the realized measures of variance but the HAR can naturally accommodate all realized measures and transformations of these measures. ${ }^{5}$. The HAR-RV model using daily, weekly and monthly ${ }^{6}$ realized volatility components may be written as follows:

$$
\sqrt{R V_{t}}=\alpha_{0}+\alpha_{d} \sqrt{R V_{t-1}}+\alpha_{w}(\sqrt{R V})_{t-5: t-1}+\alpha_{m}(\sqrt{R V})_{t-22: t-1}+u_{t}
$$

The error term $u_{t}$ is chosen to fit the distribution of the residuals, but could very well be modeled as a GARCH error (see Corsi et al. (2008), Bollerslev et al. (2009)). Since the logarithmic transformation exhibits superior forecasting performance (Andersen et al. (2007)),

\footnotetext{
${ }^{4}$ Empirical evidence of this hypothesis can be found in early contribution such as ABDL (2001a and b, 2003). Similar evidence for foreign exchange rates, futures markets, crude oil futures and the FTSE index may be found in Pong et al. (2004), Thomakos and Wang (2003), Wang et al. (2008) and Areal and Taylor (2002), respectively. ${ }^{5}$ Forsberg and Ghysels (2007) and Ghysels and Sohn (2009), note that other power transformations may be used to model the dynamics of the realized volatility. These studies show that for a number of stochastic volatility processes used in the financial literature the absolute value of the realized volatility is a better predictor of the future realized volatility, particularly for longer horizons. We do not follow this approach here.

${ }^{6}$ The optimal lag structure for the HAR model has been investigated in Craioveanu and Hillebrand (2010) who find that the genuine structure suggested in Corsi (2009) performs the best.
} 
we retain the following specification:

$$
\begin{aligned}
\log \left(R V_{t}\right)= & \omega+\phi_{1} \log \left(R V_{t-1}\right)+\phi_{2} \log \left(R V_{t-5,5}\right) \\
& +\phi_{3} \log \left(R V_{t-22,22}\right)+\gamma \epsilon_{t-1}+\eta v_{t} v_{t} \sim N I D(0,1)
\end{aligned}
$$

The error term in the volatility equation is assumed to follow a standard Gaussian, as it is well-known since Andersen et al. (2001) that the logarithmic transformation of the realized volatility is normally distributed. The latter specification captures asymmetries coming from two distinct sources: leverage effects (through $\gamma$ ), and unconditional asymmetry (with the mixture of normals).

Densities forecasts using intraday futures will be compared with forecasts obtained with the traditional EGARCH model based on daily data. The EGARCH model considered is the standard specification by Nelson (1991):

$$
\begin{aligned}
& r_{t}=\mu+\sigma_{t} \epsilon_{t}, \epsilon_{t} \sim \operatorname{NID}(0,1) \\
& \log \left(\sigma_{t}^{2}\right)=\omega+\beta \log \left(\sigma_{t-1}^{2}\right)+\gamma u_{t-1}+\xi\left|\epsilon_{t-1}\right|
\end{aligned}
$$

To make comparisons easier, a leverage term is introduced in the volatility equation. Depending on the standardized return innovation $u_{t}$ in the return equation, the volatility is impacted through the coefficient $\gamma$. The estimated value of this coefficient is generally found to be negative. Indeed, an unexpected fall in returns translates into a positive impact on the level of volatility. ${ }^{7}$

\subsection{Parameters estimation of the time series models}

The models presented in Section 2.1 are similar to those presented in Maheu and McCurdy (2011). As in their case, the estimation is performed by maximum likelihood: the estimation is possible as both the returns and the volatility are observed. ${ }^{8}$ On top of that - and in a similar fashion to Maheu and McCurdy (2011) - we assume that $\epsilon_{t}$ and $v_{t}$, that is the disturbances respectively associated to the returns and volatility, are uncorrelated. ${ }^{9}$ As it is well-known since Bertholon et al. (2006), the mixture of normals yields to estimates by QML which are very close to the true distribution.

We discuss rapidely the estimation of the model presented at equation $(3)-(12,13)$. Let $\tilde{r_{t}}$ be

\footnotetext{
${ }^{7}$ Note that the model does not allow the asymmetry to propagate into future volatility as in the EGARCH model. ${ }^{8}$ As in Maheu and McCurdy (2011), we do not resort to Monte-Carlo simulations, which would be too computationally demanding in terms of numerical implementation, particularly in a rolling window setting that we adopt for the out-of-sample forecasting comparison.

${ }^{9}$ The assumption of conditional independence does not lead to unconditional independence as the two equation are related through the leverage term. A more complete model allowing for conditional dependence has been developed in Bollerslev et al. (2009).
} 
the ex-jump return, that is

$$
\tilde{r_{t}}=r_{t}-\sum_{i=0}^{N_{t}} x_{i, t} .
$$

Let $\Omega_{t}$ be vector containing the following three processes:

$$
\Omega_{t}=\left(\tilde{r}_{t}, \sigma_{t}, J_{t}\right)
$$

where $J_{t}$ is the jump component, that is $\sum_{i=0}^{N_{t}} x_{i, t}$. The estimation of the parameters driving the joint behavior of the three processes can be obtained by maximizing their joint likelihood. The conditional joint density given the past observation of $\Omega_{t}$ can be writen as follow:

$$
\begin{aligned}
f\left(\tilde{r}_{t}, \sigma_{t}, J_{t} \mid \Omega_{t-1}\right) & =f\left(\tilde{r}_{t}, J_{t} \mid \Omega_{t-1}, \sigma_{t}\right) g\left(\sigma_{t}, J_{t} \mid \Omega_{t-1}\right) \\
& =f\left(\tilde{r}_{t} \mid \Omega_{t-1}, \sigma_{t}\right) g\left(\sigma_{t}, J_{t} \mid \Omega_{t-1}\right) h\left(J_{t} \mid \Omega_{t-1}, \sigma_{t}\right) .
\end{aligned}
$$

In equation (20)-(21), $f(),. g($.$) and h($.$) are marginal densities. Equation (21) is obtained as$ $\tilde{r}_{t}$ and $J_{t}$ are assumed to be independant. The maximization of the joint loglikelihood then clearly amounts to maximizing each of its three components independently, given that they do not share common parameters.

Maximum likelihood estimates are thus obtained through the following expressions:

$$
\begin{gathered}
\max _{\theta_{\tilde{r}}} \sum_{t=1}^{T} \log f\left(\tilde{r}_{t} \mid \Omega_{t-1}, \sigma_{t}\right) \\
\max _{\theta_{\sigma}} \sum_{t=1}^{T} \log g\left(\sigma_{t}, J_{t} \mid \Omega_{t-1}\right) \\
\max _{\theta_{J}} \sum_{t=1}^{T} \log h\left(J_{t} \mid \Omega_{t-1}, \sigma_{t}\right) .
\end{gathered}
$$

As jumps are i.i.d. random variables, it is possible to obtain closed form expressions for the model's parameters estimates:

$$
\begin{aligned}
& \hat{\lambda}=\frac{1}{T} \sum_{t=1}^{T} N_{t} \\
& \hat{\mu}_{x}=\frac{1}{\sum_{t=1}^{T} N_{t}} \sum_{t=1}^{T} \sum_{i=0}^{N_{t}} x_{i, t} \\
& \hat{\sigma}_{x}^{2}=\frac{1}{\sum_{t=1}^{T} N_{t}} \sum_{t=1}^{T} \sum_{i=0}^{N_{t}}\left(x_{i, t}-\hat{\mu}_{x}\right)^{2} .
\end{aligned}
$$


Such a straightforward estimation approach is made possible by two key elements: first, the fact that the volatility $\sigma_{t}$ is included within the filtration at time $t$; second, we assume that $\sum_{i=0}^{N_{t}} x_{i, t}$ is observable and unrelated to the other components in the dynamics of $r_{t}$. With such an approach, we obtain a realistic split between the contributions of jumps and those of volatility to the evolutions of $r_{t}$.

\subsection{Density forecasting power comparison}

To compare the various models presented earlier, we rely on out-of-sample density forecasting exercises. This subsection presents the empirical approach that we retained.

To compare density forecasts between the standard EGARCH model and the bivariate model of daily returns and HAR, we use a criteria known as the predictive likelihood (or logarithmic score). The average predictive likelihood over the out-of-sample observations $t=$ $\tau+k_{\max }, \ldots, T-k$ is:

$$
D_{M, k}=\frac{1}{T-\tau-k_{\max }+1} \sum_{t=\tau+k_{\max }-k}^{T-k} \log f_{M, k}\left(r_{t+k} \mid \Phi_{t}, \theta\right), \quad k \geq 1
$$

with $f_{\mathcal{M}, k}\left(x \mid \Phi_{t}, \theta\right)$ the $k$-period ahead predictive density for model $\mathcal{M}$, given $\Phi_{t}$ and parameter $\theta$, evaluated at the realized return $x=r_{t+k}$. Therefore, better forecasts will translate into larger $D_{\mathcal{M}, k}$.

We use a rolling window scheme to evaluate the predictive power of our forecasts. As in Maheu and MacCurdy (2011), compute the 1 to 60 day-ahead forecasts for each window. We thus obtain $T-\tau-k_{\max }+1$ data blocks for each asset. For each block, we compute the predictive likelihoods and then average over all blocks.

To evaluate the relative accuracy of competing forecasts, we rely on the test statistics developed by Diebold and Mariano (1995) in the context of the comparison of density forecasts (Amisano and Giacomini, 2007). The null hypothesis is that predictive likelihood forecasts of horizon $h$ and models $\mathcal{A}$ and $\mathcal{B}$ have the same performance. The test statistics is given by:

$$
t_{\mathcal{A}, \mathcal{B}}^{k}=\frac{\left(D_{\mathcal{A}, k}-D_{\mathcal{B}, k}\right)}{\frac{\left(\hat{\sigma}_{\mathcal{A}, \mathcal{B}, k}\right)}{\sqrt{T-\tau-k_{\max }+1}}}
$$

which is asymptotically standard normal. As for the interpretation of the Diebold and Mariano (1995) (DM) test, a significant positive (negative) estimated value rejects the null of equal performance between competing forecasts, and provides evidence in favor of model $\mathcal{A}(\mathcal{B})$. 
An additional issue arises when computing the Amisano and Giacomini (2007)'s test statistics when the jumps are explicitly incorporated within the data generating process of $r_{t}$. Indeed, as future jumps are unobservable, we need to compute the conditional distribution of $r_{t+h}$ conditionally upon the information available at time $t$.

$$
f\left(r_{t+h} \mid \mathcal{F}_{t}\right)=\sum_{i=0}^{\infty} f\left(r_{t+h} \mid \mathcal{F}_{t}, N_{t+h}=i\right) \times P\left(N_{t+h}=i\right),
$$

where $P\left(N_{t+h}=i\right)$ is the probability that the number of jumps between the date $t+h-1$ and $t+h$ is equal to $i$, given the estimated parameters. Given the estimated values for the average number of jumps by working day, we approximate the previous quantity by truncating the previous infinite sum. We compute it from $i=0$ to 10 . Beyond this threshold, the results of the numerical tests remain qualitatively unaffected. In the various HAR-based models considered here, $f\left(r_{t+h} \mid \mathcal{F}_{t}, N_{t+h}=i\right)$ is computed as in Maheu and McCurdy (2011) and in the previously mentioned cases that are not based on an explicit modeling of jumps. We also follow Maheu and McCurdy (2011) for the simulations, computations, and the use of the Newey-West long-run variance (HAC) again to make our results comparable with theirs.

\section{Empirical Results}

In this section, we present first the data used. Then, we detail the procedures used to find the optimal sampling frequency when using intraday data and to extract jumps. Finally, we comment in details the results obtained for all types of models (with/without jumps), along with the comparison of density forecasts.

\subsection{Dataset}

In our empirical analysis, we use tick-by-tick data from three different classes of assets: stock index futures, energy futures, and exchange rate. While all assets are very liquid (and are therefore suitable for using realized estimators), we need to remove days where the trading activity has not been sufficient to compute these estimators. To this end, we filter our time series with respect to three parameters: the length of the trading period in the day, the number of zero-returns and the number of transactions. Let us describe below each asset considered in the empirical analysis, and provide more details about the cleaning procedure.

The S\&P 500 futures data is a very liquid contract traded on the CME which is a tradable asset in opposition with the underlying S\&P 500 cash index. The time span for the S\&P 500 futures is from January 1, 1996 to July 31, 2008 (3,192 trading days originally). As is usual, we consider the continuous series of the front month contract using a rollover procedure which selects the 
largest volume each day to jump from one contract to the next. ${ }^{10}$. Trading of the S\&P 500 futures contract occurs from 8:30 AM to 3:15 PM which should, on normal days, provide 81 intraday returns when using a sampling interval of 5 minutes. ${ }^{11}$ We remove days with less than 81 returns which is generally an indication of part closure of the trading place. We check that all accepted days have a sufficient number of transactions, and a limited number of zero-returns as well. After cleaning the data, we obtain a sample of 3,135 days. For these selected days, the average number of trades for the continuous rollover series is 3,090 (the total number of ticks is equal to $9,809,697)$. We observe a relative stability of the number of transactions each day during the whole period.

Our second asset is the West Texas Intermediate (WTI) light sweet crude oil futures contract, traded on the New York Mercantile Exchange (NYMEX) now a branch of CME. The rollover series is built as explained previously for the $\mathrm{S} \& \mathrm{P} 500$. The period considered is from October 8, 2001 to January 15, 2010. The WTI contract is one of the most traded futures contract in the world. The total number of ticks for the continuous time series of the front month contract is equal to $52,099,419$. The trading period for the WTI futures is from 9:00 AM to 2:30 PM, which should provide 60 intraday returns each day (54 intraday returns for the September 2001/January 2007 period where trading began at 10:00 AM). Similarly to the previous asset, we remove days with less than 60 (54) intraday returns, days with more than 15 zero-returns, and days with less than 700 registered ticks. The number of observations is therefore reduced from 2,140 days to 2,081 days when all these requirements are met. The mean number of trades is equal to 25,035 . In contrast to S\&P 500 futures, this figure is very different before and after mid-2006, which is mainly due to the launch of electronic trading. ${ }^{12}$

The USD/JPY exchange rate, the third asset, covers the period going from December 30, 1996 to June 1,2007 , i.e. a sample of 2,701 days (2,720 initially before cleaning the data) with a total of 22,530,929 transactions (8,342 ticks per day on average). As in previous contributions (see Andersen et al. (2001) among many others), we consider the period going from 9:00 PM to 8:59 PM the next day as a unit period, because the FX market is opened on a 24-hour window.

\section{Insert Figure 1}

Figure 1 displays the time-series of S\&P 500 futures, WTI futures, and the USD/JPY exchange rate, along with the open-to-close log-returns. ${ }^{13}$ As may be seen from these plots, we include in our analysis time-series with very different properties: more or less pronounced volatility clustering, bear and bull markets, extreme variations in a short period of time, etc.

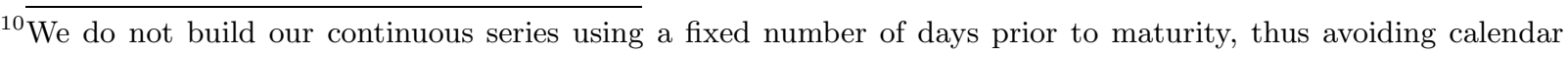
effects.

${ }^{11}$ We discuss the issue of sampling frequency in the next section.

${ }^{12}$ We have an average number of 2,214 ticks per day during the September 4, 2001 / August 31, 2006 period vs. 57,054 ticks for the September 1, 2006 / January 15, 2010 period.

${ }^{13}$ We choose to work with open-to-close returns because overnight returns have shown to follow a very different dynamics. In addition, including overnight returns may alter our analysis when standardizing returns as we work with volatility computed with intraday transaction data.
} 


\section{Insert Table 1}

We also present summary statistics for all time series in Table 1 . We observe that the realized volatility and the bi-power variation measures present nonzero skewness and excess kurtosis. ${ }^{14}$ These descriptive statistics therefore reveal a "fat tailed" distribution. The logarithm transformations of these quantities are nearly Gaussian, which is a common finding since Andersen et al. (2001) among others. ${ }^{15}$

\subsection{Optimal sampling frequency and jump detection}

This subsection presents empirical technicalities regarding the intra-day volatility and jumps measures: (i) the determination of the optimal sampling frequency for each asset, (ii) the jump detection, and (iii) the sequential jump detection procedures.

\subsubsection{Optimal sampling frequency}

For these three estimators of realized volatility, theory suggests that returns should be computed at the highest possible frequency, so that estimators converge asymptotically towards the true conditional volatility. However, it is well-known since Andersen and Bollerslev (1997, 1998) and Taylor and Xu (1997) that microstructure noise (due to price discreteness, bid-ask spread, non-synchronous trading, etc. $)^{16}$ may impact the realized volatility estimator at high frequency.

To deal with this issue while making our results comparable with the rest of the literature, we follow the 5 minutes 'rule-of-thumb'. As our three series are highly liquid assets, this sampling interval is adequate to make our realized measures not to be impacted by the noise.

\section{Insert Figure 2}

\section{Insert Figure 3}

We examine further this question for the WTI crude oil futures price series, which did not benefit from such an analysis in previous research. In Figures 2 and 3, we report the volatility signature plot for oil futures. This analysis is crucial as the trading activity dramatically increased in

\footnotetext{
${ }^{14}$ Note for a normally distributed random variable skewness is zero, and kurtosis is three.

${ }^{15}$ We come back on this issue when modeling the volatility using the log transformation. Goncalves and Meddahi (2011) suggest a new class of nonlinear transformations based on the Box-Cox transformation which outperform the log transformation in Monte Carlo simulations. We leave as an extension this possible transformation and follow the bulk of the empirical literature by considering the logarithm.

${ }^{16}$ See Hansen and Lunde (2006) for a thorough discussion of this issue and Andersen et al. (2011) for a theoretical and empirical analysis of the impact of microstructure noise on the forecast of realized volatility. To deal with this issue, we use staggered versions of BPV and MedRV as advocated in Huang and Tauchen (2005).
} 
September 2006 following the generalization of electronic trading (see Section 3.1) and may result in different noise structure before and after this event. These graphs confirm that the standard 5-minute sampling frequency seems to be appropriate in this case as well.

\subsubsection{Jump detection}

Once the optimal sampling frequency is determined, realized volatility, bipower variation and median realized volatility estimators are computed. The difference between $R V$ and a jumprobust estimator such as BPV or MedRV provides, when it is statistically significant, an estimate of the sum of squared jumps $\sum_{j=1}^{J(t)} \kappa^{2}\left(t_{j}\right)$ which have occurred during the period under investigation. Note that a small estimated value for a jump may not be actually a jump but a variation due to the continuous path of the stochastic process and the presence of a jump has thus to be formally tested. BNS $(2004,2006)$ develop such a testing framework using asymptotic theory on realized variance and multipower variations. ${ }^{17}$

As Andersen et al. (2007) put it, "significant" jumps may be identified by comparing realizations of test statistics to a standard normal distribution. They use the test statistic by Huang and Tauchen (2005) to examine the significance of a jump when the chosen jump-robust estimator is the BPV:

$$
Z J_{B P V}(N, d)=\sqrt{N} \frac{\left(R V_{d, N}-B P V_{d, N}\right) R V_{d, N}^{-1}}{\left(\left(\xi_{1}^{-4}+2 \xi_{1}^{-2}-5\right) \max \left\{1, T Q_{d, N} B P V_{d, N}^{-2}\right\}\right)^{1 / 2}}
$$

with $T Q$ the realized tripower quarticity, which converges in probability to the integrated quarticity. The ratio-statistic in equation (28) has reasonable power against several empirically realistic calibrated stochastic volatility jump diffusion models (Andersen et al., 2007).

The test may be adapted to the MedRV estimator as follows:

$$
Z J_{M e d R V}(N, d)=\sqrt{N} \frac{\left(R V_{d, N}-M e d R V_{d, N}\right) R V_{d, N}^{-1}}{\left(0.96 \max \left\{1, M e d R Q_{d, N} M e d R V_{d, N}^{-2}\right\}\right)^{1 / 2}}
$$

with $M e d R Q$ an estimate of the integrated quarticity obtained by using the same methodology as for MedRV. Theodossiou and Zikes (2009) show by means of many simulations and empirical analysis that this test has better properties in the presence of jumps of finite or even infinite activity and zero-returns. ${ }^{18}$ If, as we will demonstrate, disentangling jumps from the

\footnotetext{
${ }^{17}$ Veraart (2010) studies the limit theory of these estimators in the presence and absence of jumps.

${ }^{18}$ Several other estimators for identifying jumps in the series, such as QRV (Christensen et al., 2010) estimator, which are shown to be more robust in the presence of microstructure noise and zero-returns, may be used. Theodossiou and Zikes (2009) provide a very complete treatment of existing jump detection tests as well as their relative performance in case of microstructure noise and/or jumps. In light of the good properties of the MedRV-based test, we focus on this alternative estimator.
} 
continuous component help in forecasting the density of returns, we are particularly interested in investigating whether the better properties of the test base on MedRV compared to BPV will translate in an improvement in density forecasting as well. A preliminary analysis ${ }^{19}$ shows that for both samples "jumpy days" are similar using MedRV instead of BPV, but that the magnitude of jumps is slightly different.

\section{Insert Figure 4}

\section{Insert Figure 5}

\section{Insert Figure 6}

Figures 4 to 6 show the realized volatility, the BPV and the jump component from BPV, as well as the MedRV and the jump component from MedRV for our four series. A preliminary analysis, not reproduced here to conserve space, but available upon request to the authors, shows that for both samples "jumpy days" are quite similar using MedRV instead of BPV, but that the magnitude of jumps is slightly different.

\section{Insert Table 2}

More interestingly, Table 2 provides statistics about the contribution of jumps to the total realized volatility for our four series and for different level of significance of the test. ${ }^{20}$ These values are in line with results in Huang and Tauchen (2005) and Andersen et al. (2007) for S\&P 500 and FX. Our results for WTI are new but very similar to the S\&P 500 futures case. Overall, these results point to the fact that jumps contribute to a significant part of the total return variation. Because it is well-known that jumps are not persistent while the continuous component is, considering jumps independently is likely to be rewarded in a forecasting exercise.

\section{Insert Table 3}

Table 3 gives the Kolmogorov-Smirnov statistics for different distributions. Results indicate that the Gaussian distribution does not provide a good fit to the series of returns standardized by realized volatility, bipower variation, and median realized volatility. Given these results (where we do not specifically model the jumps), we assume that the conditional distribution of returns is a mixture of Gaussian distributions, in a manner closely related to Maheu and McCurdy (2011).

\footnotetext{
${ }^{19}$ Not reproduced here to conserve space, but available upon request to the authors.

${ }^{20}$ The "no threshold" case corresponds to the case measure of jump contribution in Huang and Tauchen (2005) where the difference between RV and BPV is taken directly.
} 


\subsubsection{Sequential jump detection}

For the purpose of modeling the jump component independently from the continuous component, we need a precise measure of jumps including their size along with their sign. We obtain such a measure using the sequential jump detection procedure developed in Andersen et al. (2010). ${ }^{21}$ The idea behind this procedure is simple. We first use the standard test presented below using either BPV or MedRV on intraday returns for day $d$. If we do not reject the existence of a jump for day $d$, we consider that the largest return in absolute value is a jump and remove it from intraday returns. We replace this "jump" return with the mean of remaining intraday returns and run the jump test again to check the presence of another jump in the same day.

\section{Insert Figure 7}

\section{Insert Figure 8}

One may wonder whether, in light of the rarity of jumps, sequential detection is useful? To answer this question we first plot in Figures 7 and 8 transactions for S\&P 500 and WTI and FX respectively. For each asset, the left panel plots a day with exactly one jump while the right panel plots a day with two or more jumps identified with the sequential procedure.

\section{Insert Table 4}

As a second, and more rigorous evidence, we provide in Table 4 statistics about the number of jumps we detect each day for all series. These statistics support the view that days with more than a single jump are quite common.

Using the sequential detection procedure with both the BPV and the MedRV procedure, we obtain for each asset a series of jumps along with their sign and size which will be used in the econometric estimation below. As a rapid check of our results, we compare squared jumps with the squared component resulting from the difference between RV and the jump-robust measure (when significant) and confirm the importance of the sequential procedure.

\subsection{Estimation Results}

As noted above, we estimate the models on a rolling window of 1,260 daily observations for all series. Concerning the average volatility level for each asset class, we obtain the expected result that the BPV and MedRV estimators are less volatile than the "naive" RV estimator (Table 1). For the S\&P500, we find that the logarithmic transformations of the volatility measures

\footnotetext{
${ }^{21} \overline{\mathrm{A}}$ quite similar procedure is developed earlier in Andersen et al. (2007b).
} 
present a distribution with thin tails. For the WTI futures and the USD/JPY exchange rate, we find evidence of leptokurticity (but globally less for $\log \left(B P V_{t}\right)$ and $\log \left(M e d R V_{t}\right)$ ). Besides, the ranking of volatilities is coherent with our economic intuition: the WTI futures contract is found to be more volatile than the S\&P500, and finally the USD/JPY exchange rate.

Concerning the Kolmogorov-Smirnov statistics for daily open-to-close standardized returns, we uncover the adequation to various distributions. The returns are clearly non-Gaussian when looking at the skewness and kurtosis statistics in Table 3. In all models, we unambiguously reject the adequation to the Gaussian law. Due to the asymmetric patterns present in the data, the Student- $t$ distribution is rejected as well. Interestingly, we accept various distributions which account for asymmetry such as the Generalized Hyperbolic and the Mixture of Gaussian distributions. In the estimation of the bivariate models, we focus on the Mixture of Normals because its parameters are easily interpretable in economic terms. In addition, its estimation is easier numerically and thus more adapted to a rolling estimation scheme.

Concerning the presence of jumps, we find for all assets a contribution of jumps to the volatility level (Tables 2 and 4). The contribution of jumps is the highest for the USD/JPY exchange rate $(14.45 \%$ with no threshold for the detection as in Huang and Tauchen (2005)), followed by the WTI futures contract (6.88\%), and finally the S\&P500 (5.24\%). In terms of frequency, the number of days with jumps is quite important, and strictly positive for all assets. The most common situation is when we observe the occurence of one jump per day. Furthermore, we note that the WTI futures contract is characterized by the highest frequency of jumps (as the percentage of days with jumps is superior to $11 \%$ ). Finally, it seems that for the USD/JPY the jumps are less frequent than for other assets (6.78\%). However, the USD/JPY and the S\&P 500 are characterized by the highest number of days with several jumps within the same day. The S\&P 500 records 82 days with more than one jump, which is roughly equal to $28 \%$ of the number of days with jumps. These ratios are equal to $29 \%$ and $44 \%$ for, respectively, the WTI futures contract and the USD/JPY exchange rate.

\section{Insert Table 5}

\section{Insert Table 6}

\section{Insert Table 7}

Moving to the estimates of the bivariate models in Tables 5 to 7 , we focus our comments on the most interesting parameters. The leverage effect varies depending on the estimates. For the WTI futures contract and the S\&P 500, $\gamma$ is closer to zero when relying on the MedRV or BPV estimators. For the USD/JPY exchange rate, we find that $\gamma$ is statistically equal to zero based on the MedRV and BPV measures. When looking at the persistence of the HAR components, we find that the $\phi_{1}, \phi_{2}$ and $\phi_{3}$ coefficients vary depending on the distributions and the measures of volatility. In the case of the WTI futures contract for instance, the $\phi_{1}$ and $\phi_{3}$ components 
lose in persistence when one moves from the RV estimator to the BPV and MedRV estimators. However, the $\phi_{2}$ component gains relatively in persistence. In the case of the S\&P 500, if we compare the HAR-MedRV-Mixture with the HAR-MedRV-Jump, we find that the persistence of the $\phi_{1}\left(\phi_{2}\right)$ component decreases (increases) when moving to the latter model. These effects can be seen as being very specific to the sample data. In addition, the mixture parameter $\phi$ also varies depending on the volatility measure used. With the WTI futures contract, we find that $\hat{\phi}=0.449$ with the RV estimator, and $\hat{\phi}=0.486$ with the BPV estimator. For the remaining parameters, we can notice a relative stability of the results obtained across Tables 5 to 7 .

Note that we can also compare the degree of activity of the jump component, i.e. it is possible to rank the assets depending on the intensity and the volatility level of jumps. In the case of the HAR-BPV-Jump model for instance, the USD/JPY exchange rate records the highest intensity of jumps $(\lambda=1.19)$, while the WTI futures contract ranks second $(\lambda=0.149)$, and the S\&P 500 exhibits the lowest intensity $(\lambda=0.138)$. Finally, the WTI futures contract exhibits the highest volatility of jumps $\left(\sigma_{x}=0.010\right)$, followed by the S\&P $500\left(\sigma_{x}=0.006\right)$ and the USD/JPY exchange rate $\left(\sigma_{x}=0.002\right)$ for the HAR-BPV-Jump model. Thus, we uncover that there are different types of jumps (i.e. either frequent and small jumps, or less frequent and large jumps) specific to each asset class considered in this article.

\subsection{Forecast accuracy}

\section{Insert Table 8}

In terms of forecast accuracy, we find that the bivariate models which take explicity into account the jumps tend to perform better than the models based on a Gaussian distribution. This comment is valid for all assets. For the S\&P 500, the BPV and MedRV Gaussian models are dominated by the RV Gaussian model. For instance, in Table 8, the Diebold-Mariano test statistic is equal to 2.878 for the Realized Gaussian vs. Bipower Gaussian models at a 30-day horizon. Besides, we confirm the result by Maheu and McCurdy (2011) that the EGARCH model is dominated by the Realized Gaussian model (with a Diebold-Mariano test statistic equal to -3.050 at the 10-day horizon). More generally, at the 5- and 10-day horizons, all non Gaussian models are equivalent, whatever the volatility measure. In Table 8 , it appears interesting to note that the Bipower Jump model beats the MedRV Jump model at all horizons. At the more distant horizons (up to 60-day), we can conclude that the Bipower Jump model dominates all other models.

\section{Insert Table 9}

When looking at the WTI futures contract, we uncover the same effects as in Maheu and McCurdy (2011), and regarding the comparison of the BPV/MedRV models compared to the Realized Gaussian model. For instance, the Diebold-Mariano test statistic is equal to 4.717 for 
the Realized Gaussian vs. Bipower Gaussian models at a 30-day horizon. At the 5-day horizon, all non Gaussian models either are equivalent or dominate Gaussian models according to the Diebold-Mariano test statistics in Table 9. For the horizons superior to 10-day, the Realized MN model dominates the Bipower MN and MedRV MN models. In addition, we note that the BPV Jump model dominates the MedRV model at all horizons.

\section{Insert Table 10}

Finally, the results of the Diebold-Mariano test statistics for the USD/JPY exchange rate are presented in Table 10. They show, by and large, that the Bipower Gaussian and MedRV Gaussian models dominate the Realized Gaussian model, which is equivalent to or preferred to the EGARCH model for any horizon superior to 30-day. The Bipower Jump and MedRV Jump models dominate all other models, while MedRV tends to dominate BPV at all horizons in that latter case.

\section{Insert Figure 9}

\section{Insert Figure 10}

\section{Insert Figure 11}

The same insights can be gathered by looking at the average predictive likelihood for the density of the three assets at various horizons (up to 60-day) in Figures 9 to 11. When looking at these graphs, recall that for the interpretation of the Diebold-Mariano test statistic, a significant positive (negative) estimated value rejects the null of equal performance between competing forecasts, and provides evidence in favor of model $\mathcal{A}(\mathcal{B})$. These graphs confirm the highly superior forecast accuracy of models based on intraday data for all of our series. This was the main result in Maheu and McCurdy (2011), and we confirm their findings for the three assets under investigation. For the S\&P 500, we observe the superiority of BPV Jump and MedRV Jump estimators for density forecasting particularly at horizons of 20 to 60 days. BPV and MedRV estimators also have good performance for the WTI in comparison with models using realized volatility estimated using the mixture of normals. This indicates that the jump component either included in the total realized volatility (using the mixture of normals) or modeled separately provides information in forecasting the density of returns. Results are very similar for the USD/JPY. The superiority of the BPV Jump and the MedRV Jump is evident at all horizons.

Overall, the empirical results obtained concerning the forecast accuracy depend on the asset under consideration, and the nature of the activity of the jump component. Departing from Maheu and McCurdy (2011), our results therefore tend to provide a deeper understanding of the effects at stake when decomposing the jump and continuous components of volatility. Namely, 
modeling explicitly jumps is of primary importance to achieve better performances with bivariate models, while decomposing between jumps and the continuous component volatility appears of secondary importance.

\section{Conclusion}

Jumps in assets' returns are to be related to strong directional variations in the prices that the market participants agree on given their evaluation of the perspectives for a given financial asset. $^{22}$ On the contrary, volatility reflects a certain lack of agreement within financial markets: as they do not lead to strong directional variations but up-and-down swings in prices, they should not be mistaken with jumps. In such cases, jumps and volatility do not receive the attention they deserve from an econometric viewpoint.

This paper has examined the forecasting power of jumps in addition, specifically, to the continuous component when the density of returns is the variable of interest. Whilst numerous authors have considered the informational content of continuous vs. jump components for volatility forecasting, none have thought to address the particular question of density forecasting. Detection of such information for different classes of assets would indicate the ability of new econometric models to anticipate the evolution of density returns in a fundamentally different way compared to more traditional forecasting models.

Our results unveil new effects regarding the importance of distinguishing between the continuous and jump components of volatility. In this regard, this article specifically extends the findings by Maheu and McCurdy (2011) by considering various bivariate models with/without jumps. The empirical application is devoted to three types of assets: the S\&P 500, the WTI futures contract, and the USD/JPY exchange rate. The main results may be summarized as follows. First, we confirm the findings by Maheu and McCurdy (2011) that intraday data yield better densities forecasts than daily data. Second, and more importantly, we assess the importance of jumps when forecasting the density of returns by comparing jump-robust (bipower variation, median realized volatility) and non-robust measures of realized volatilities. Compared to the "naive" measure of realized volatility, considering jumps specifically provides significant improvement on the accuracy of forecasts of return densities.

Two central concluding remarks arise. First, we have shown in this paper that the explicit modelling of jumps is central in the estimation of bivariate models in the fashion of Maheu and McCurdy (2011). Such an explicit modeling task yields better performances, as shown in our empirical application and with the forecast accuracy tests of all competing models. Second, discriminating between the continuous component of volatility and jumps appears comparatively less important across our estimates. From that perspectives, our results can be seen as an extension of the contribution by Maheu and McCurdy (2011).

\footnotetext{
${ }^{22}$ Lahaye et al. (2011) and Evans (2011) have related news to the occurrence of jumps in intraday data. Nevertheless, some jumps are not related to macro announcements or news and may be the consequence of microstructure effects.
} 
Other jump detection techniques may be used (see Boudt et al. (2011), or Christensen et al. (2010) among others). Boudt et al. (2011) in particular provide very interesting empirical results because their measure take the intraday periodicity into account and thus does not over-detect jumps in low-volatility periods and does not under-detect jumps in periods of high-volatility. Nevertheless, collectively taken, our results are sufficiently strong so that we can believe they would be robust to alternative jump detection methods. 


\section{References}

Amisano, G., Giacomini, R., 2007. Comparing density forecasts via weighted likelihood ratio tests. Journal of Business and Economic Statistics 25, 177-190.

Andersen, T. G., Bollerslev, T., 1997. Intraday periodicity and volatility persistence in financial markets. Journal of Empirical Finance 4, 115-158.

Andersen, T.G., Bollerslev, T., 1998. Answering the skeptics: yes, standard volatility models do provide accurate forecasts. International Economic Review 39, 885-905.

Andersen, T.G., Bollerslev, T., Diebold, F.X., 2007. Roughing it up: including jump components in the measurement, modeling and forecasting of return volatility. Review of Economics and Statistics 89, 701-720.

Andersen, T.G., Bollerslev, T., Diebold, F.X., Ebens, H., 2001. The distribution of stock return volatility. Journal of Financial Economics 61, 43-76.

Andersen, T.G., Bollerslev, T., Diebold, F.X., Labys, P., 2001. The distribution of exchange rate volatility. Journal of the American Statistical Association 96, 42-55.

Andersen, T.G., Bollerslev, T., Diebold, F.X., Labys, P., 2003. Modeling and forecasting realized volatility. Econometrica 71, 579-625.

Andersen, T.G., Bollerslev, T., Dobrev, D., 2007. No-arbitrage semi-martingale restrictions for continuoustime volatility models subject to leverage effects, jumps and i.i.d. noise: Theory and testable distributional implications. Journal of Econometrics 138, 125-180.

Andersen, T.G., Bollerslev, T., Huang, X., 2010. A semiparametric framework for modeling and forecasting jumps and volatility in speculative prices. Journal of Econometrics, forthcoming.

Andersen, T.G., Bollerslev, T., Meddahi, N., 2011. Realized volatility forecasting and market microstructure noise. Journal of Econometrics 160, 220-234.

Andersen, T.G., Dobrev, D., Schaumburg, E.,, 2009. Jump-robust volatility estimation using nearest neighbor truncation. NBER Working Paper 15533, Cambridge, MA.

Areal, N., TAYlor, S.J., 2002. The realized volatility of FTSE-100 futures prices. Journal of Futures Markets $22,627-648$.

Barndorff-Nielsen, O., Shephard, N., 2002. Econometric analysis of realized volatility and its use in estimating stochastic volatility models. Journal of the Royal Statistical Society, Series B 64, 253-280.

Barndorff-Nielsen, O., Shephard, N., 2004. Power and bipower variation with stochastic volatility and jumps. Journal of Financial Econometrics 2, 1-37.

Barndorff-Nielsen, O., Shephard, N., 2006. Econometrics of testing for jumps in financial economics using bipower variation. Journal of Financial Econometrics 4, 1-30.

Bertholon, H., Montfort, A., Pegoraro, F., 2006. Pricing and inference with mixtures of conditionally normal processes. Crest DP, Banque de France Working Paper \# 188.

Bollerslev, T., Kretschmer, U., Pigorsch, C., Tauchen, G., 2009. A discrete-time model for daily S\&P500 returns and realized variations: jumps and leverage effects. Journal of Econometrics 150, 151-166.

Boudt, K., Croux, C., Laurent, S., 2011. Outlyingness weighted quadratic covariation. Journal of Financial Econometrics, forthcoming.

Christensen, K., Oomen, R., PodolskiJ, M., 2010. Realised quantile-based estimation of the integrated variance. Journal of Econometrics 159, 74-98.

Clements, M.P., 2005. Evaluating Econometric Forecasts of Economic and Financial Variables. Basingstoke and London: Palgrave Macmillan.

Corradi, V., Swanson, N.R., 2006. Predictive density evaluation. In: G. Elliot, C.W.J. Granger and A. Timmermann (Eds.), Handbook of Economic Forecasting, vol. 1, Elsevier.

Consi, F., 2009. A simple approximate long memory model of realized volatility. Journal of Financial Econometrics 7, 174-196.

Corsi, F., Mittnik, S., Pigorsch, C., Pigorsch, U., 2008. The volatility of realized volatility. Econometric Reviews 27, 46-78.

Corsi, F., Pirino, D., Renò, R., 2010. Threshold bipower variation and the impact if jumps on volatility 
forecasting. Journal of Econometrics 159, 276-288.

Craioveanu, M., Hillebrand, E., 2010. Why it is O.K. to use the HAR-RV $(1,5,21)$ model. Unpublished manuscript.

Diebold, F.X., Mariano, R.S., 1995. Comparing Predictive Accuracy. Journal of Business E Economic Statistics 13, 253-263.

Evans, K.P., 2011. Intraday jumps and US macroeconomic news announcements. Journal of Banking and Finance, forthcoming. doi: 10.016/j.jbankfin.2011.02.018

Forsberg, L., Ghysels, E., 2007. Why do absolute returns predict volatility so well. Journal of Financial Econometrics 5, 31-67.

Ghysels, E., Sohn, B., 2009. Which power variation predicts volatility well? Journal of Empirical Finance 16(4), 686-700.

Goncalves, S., Meddahi, N., 2011. Box-Cox transforms for realized volatility. Journal of Econometrics 160(1), 129-144.

HAnsen, P.R., Huang, Z., Shek, H.H., 2011. Realized GARCH: a joint model for returns and realized measures of volatility. Journal of Applied Econometrics, in press.

HAnsen, P.R., Lunde, A., 2006. Realized variance and market microstructure noise. Journal of Business and Economic Statistics 24, 127-218.

Huang, X., Tauchen, G., 2005. The relative price contribution of jumps to total price variance. Journal of Financial Econometrics 3, 456-499.

Lahaye, J., Laurent, S., Neely, C.J., 2011. Jumps, cojumps, and macro announcements. Journal of Applied Econometrics, forthcoming. doi: 10.1002/jae.1149

Liu, C., Maheu, J., 2009. Forecasting Realized Volatility: A Bayesian Model-Averaging Approach. Journal of Applied Econometrics 24, 709-733.

Maheu, J.M., McCurdy, T.H., 2011. Do high-frequency measures of volatility improve forecasts of return distributions? Journal of Econometrics 160, 69-76.

Muller, U.A., Dacorogna, M.M., Dave, R.D., Olsen, R.B., Pictet, O.V., 1997. Volatilities of different time resolutions - Analyzing the dynamics of market components. Journal of Empirical Finance 4, 213-239.

Pong, S., Shackleton, M.B., Taylor, S.J., Xu, X., 2004. Forecasting currency volatility: a comparison of implied volatilities and AR(FI)MA models. Journal of Banking and Finance 28, 2541-2563.

Shephard, N., Sheppard, K., 2010. Realising the future: forecasting with high-frequency-based volatility (HEAVY) models. Journal of Applied Econometrics 25(2), 197-231.

TAY, A.S., WAlLis, K.F., 2000. Density forecasting: a survey. Journal of Forecasting 19, 235-54.

TAYLOR, S.J., Xu, X., 1997. The incremental volatility information in one million foreign exchange quotations. Journal of Empirical Finance 4, 317-340.

Theodosiou, M., Z̆ıKes̆, P., 2011. A comprehensive comparison of alternative tests for jumps in asset prices. Working Paper 2011-2, Central bank of Cyprus, July.

Thomakos, D.D., Wang, T., 2003. Realized volatility in the futures markets. Journal of Empirical Finance $10,321-353$.

Timmermann, A., 2006. Forecast combinations. In Elliott, G., Granger, C.W.J. and Timmermann, A. (Eds.), Handbook of Economic Forecasting Volume 1, 135-96. Amsterdam: North-Holland.

VERAART, A.E.D., 2010. Inference for the jump part of quadratic variation of Ito semimartingales. Econometric Theory 26(2), 331-368.

WALlis, K.F., 2007. Forecast uncertainty, its representation and evaluation. In Mariano, R.S., Tse, Y.K. (Eds.), Econometric Forecasting and High-Frequency Data Analysis Volume 13, 1-51. Lecture Notes Series of the Institute for Mathematical Sciences, National University of Singapore. Singapore: World Scientific.

WANG, T., Wu, J., YANG, J., 2008. Realized volatility and correlation in energy futures markets. Journal of Futures Markets 28, 993-1011. 


\section{Figures}

Figure 1: Raw time-series (left panel) and open-to-close standardized log-returns (right panel)
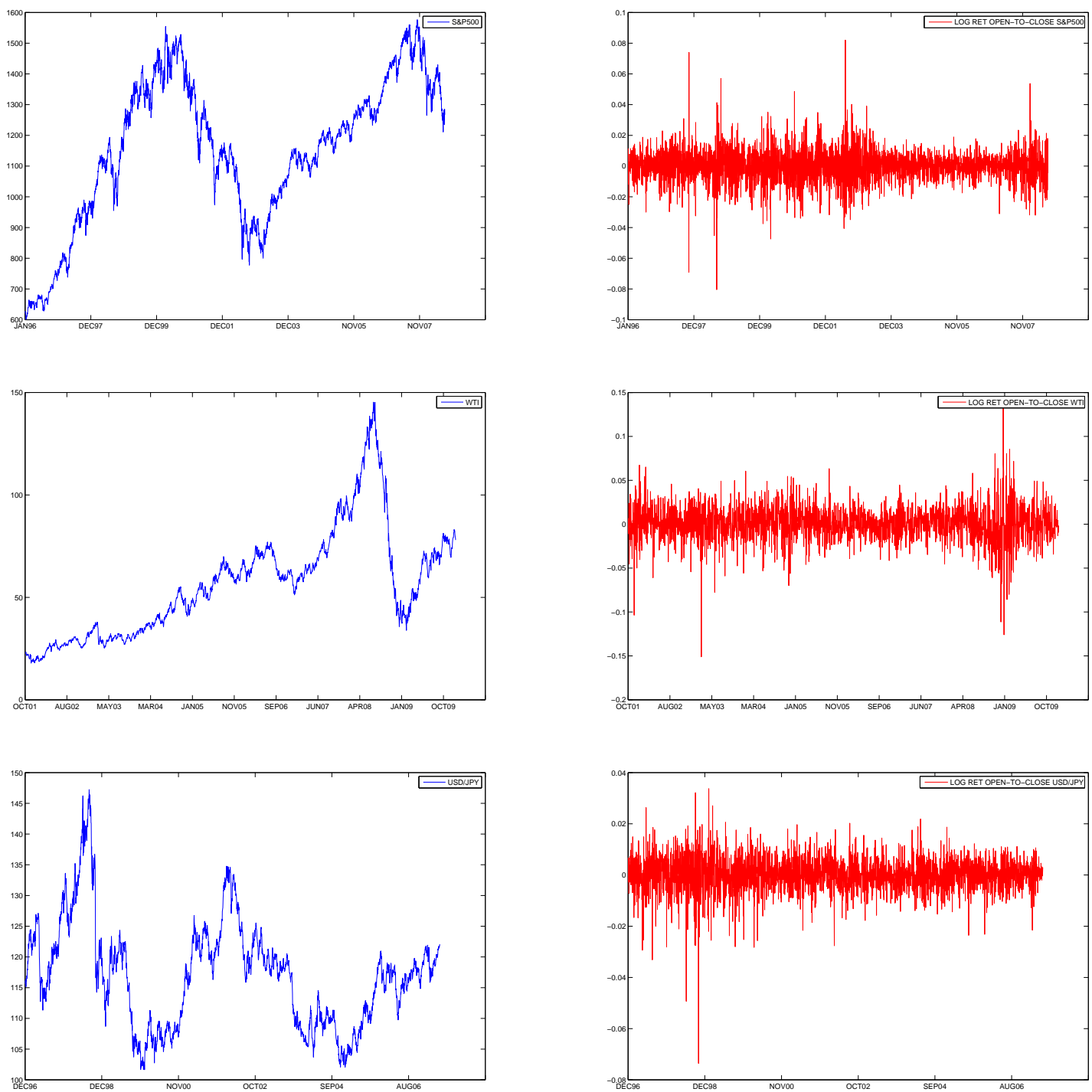
Figure 2: Volatility signature plot for the oil futures contract using front month rollover and the realized volatility, bipower variation and median realized estimators (2001-2006).

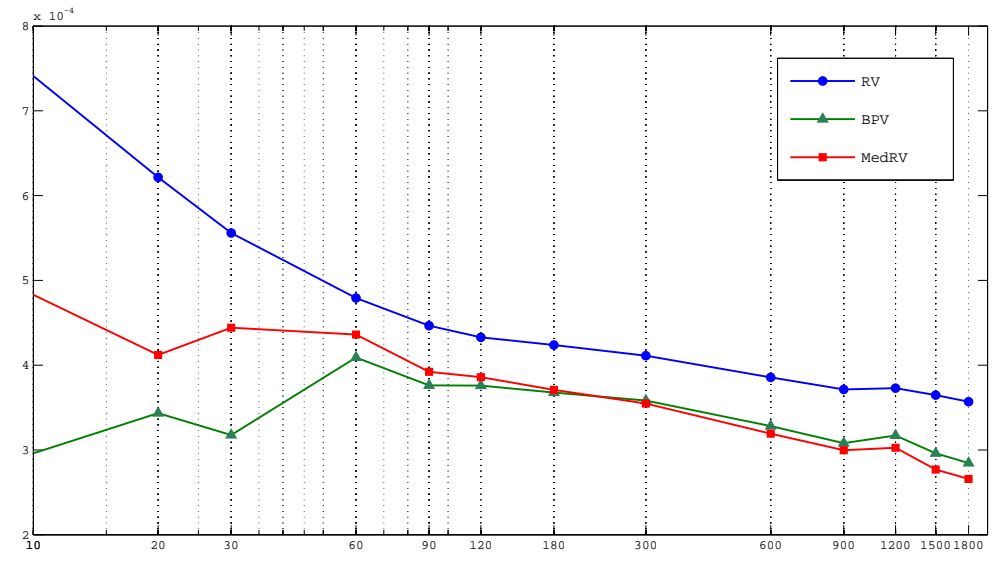

Figure 3: Volatility signature plot for the oil futures contract using front month rollover and the realized volatility, bipower variation and median realized estimators (2006-2010).

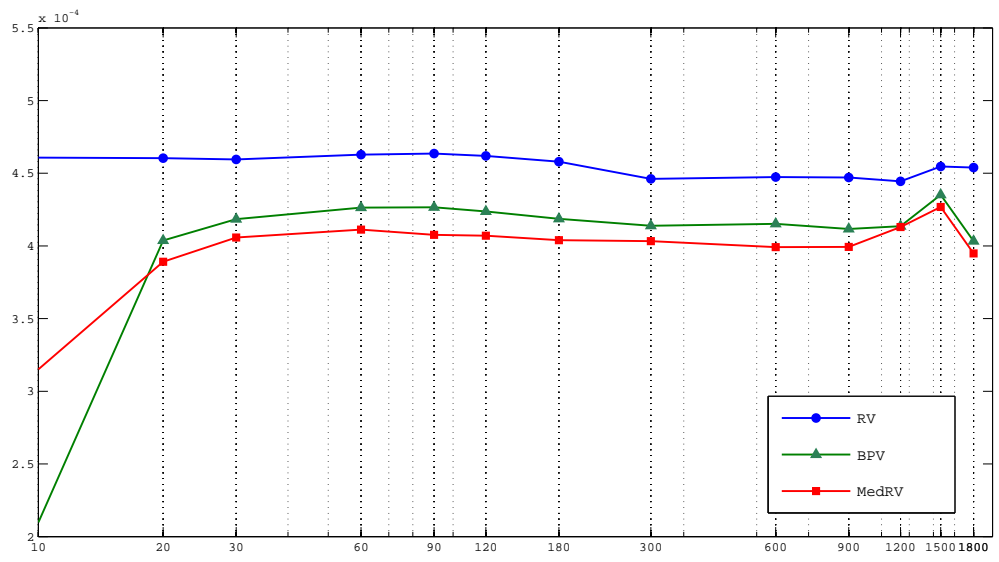


Figure 4: Realized volatility, bi-power variation with jump component, and median realized volatility with jump component for S\&P500 futures (from top to bottom and left to right).
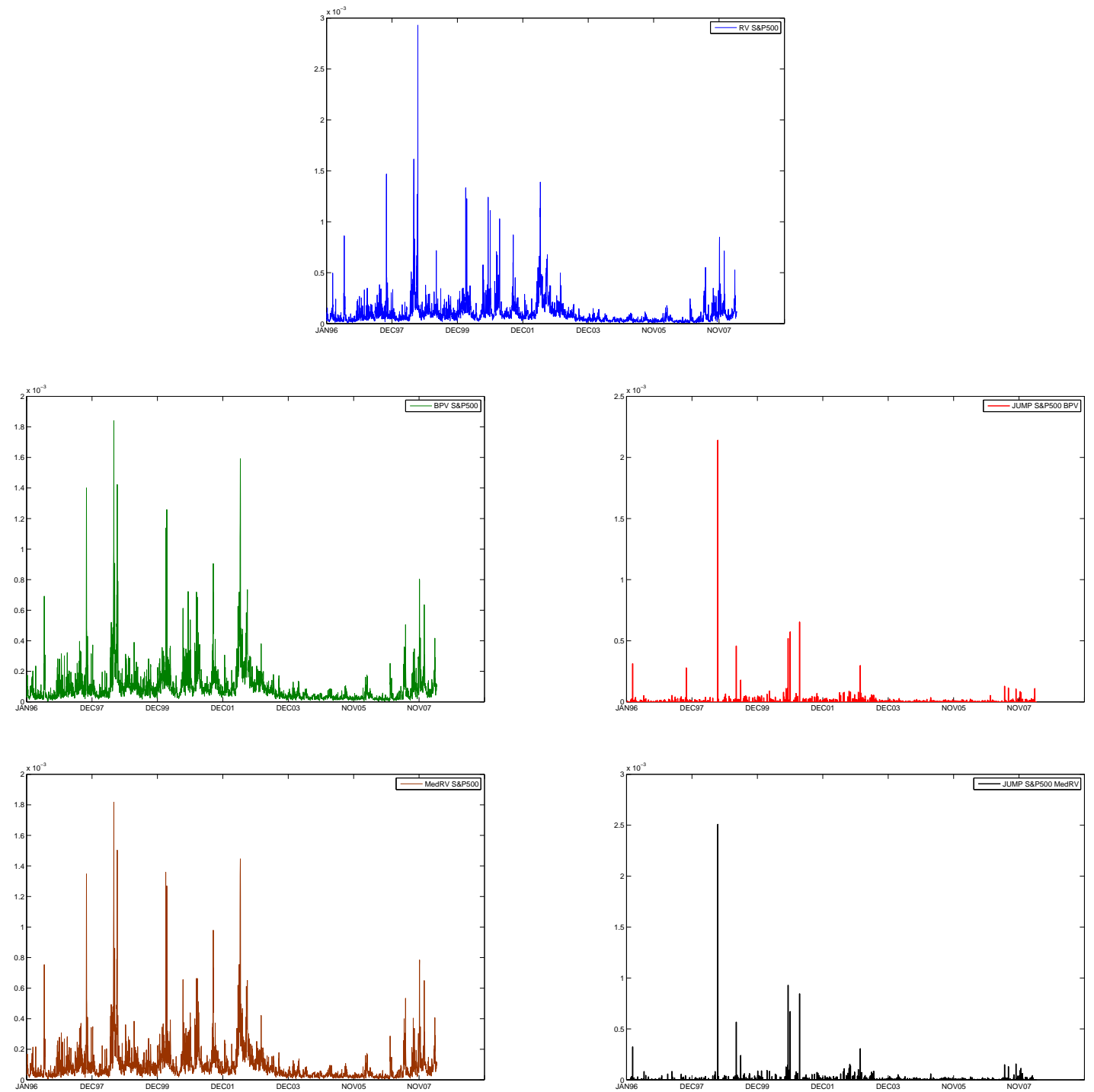
Figure 5: Realized volatility, bi-power variation with jump component, and median realized volatility with jump component for WTI futures (from top to bottom and left to right)
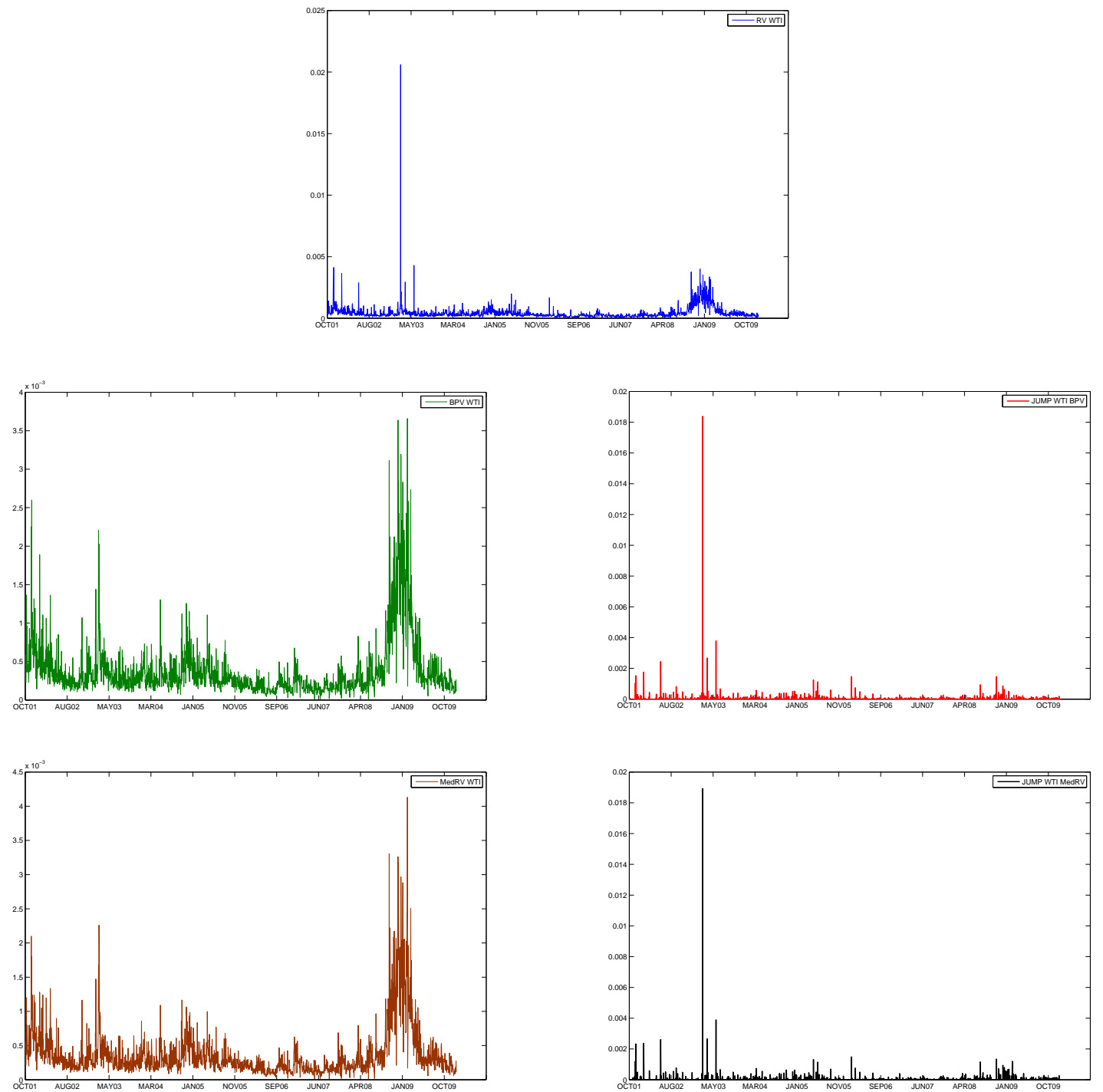
Figure 6: Realized volatility, bi-power variation with jump component, and median realized volatility with jump component for USD/JPY (from top to bottom and left to right).
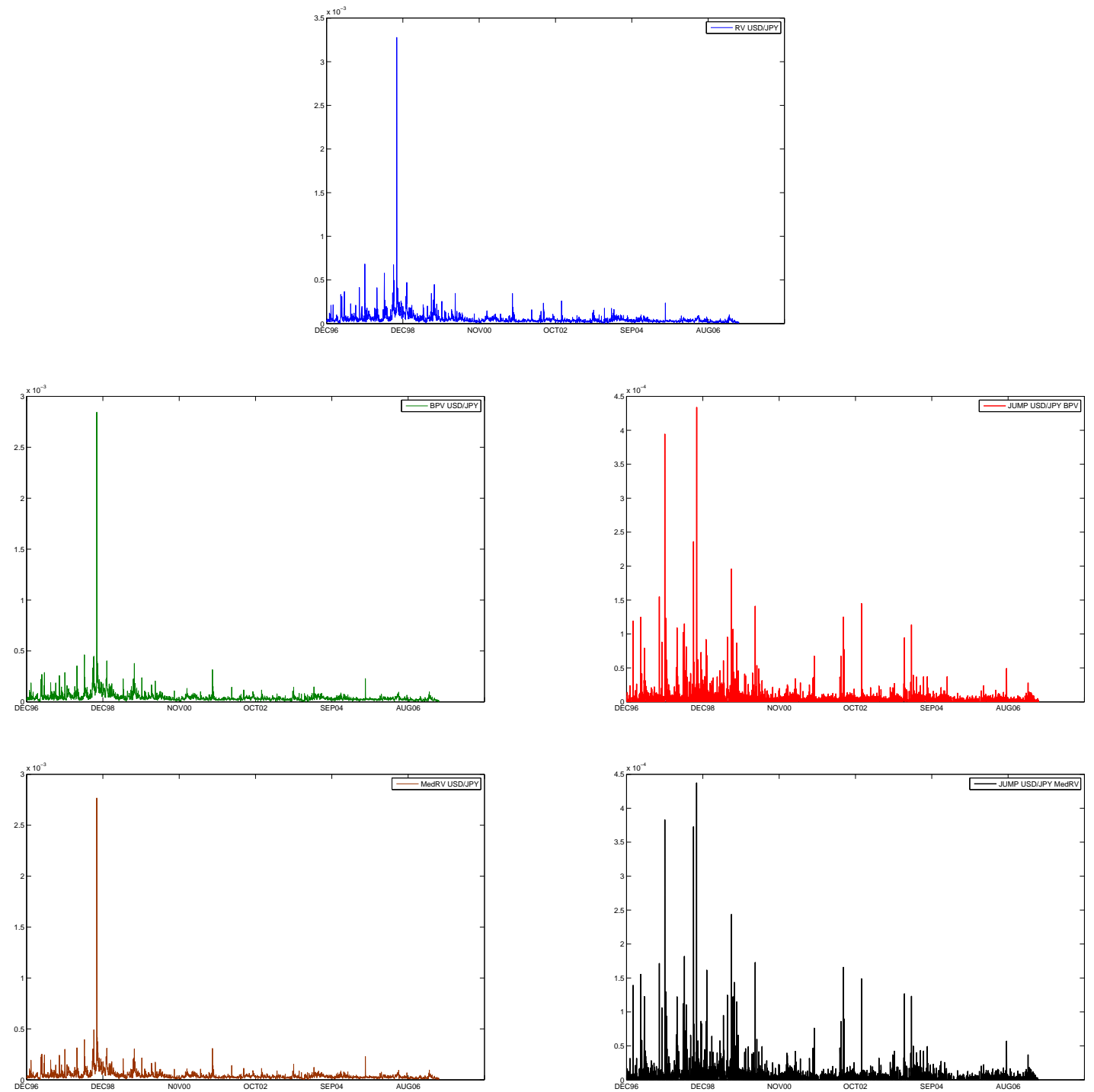
Figure 7: Jumpy days
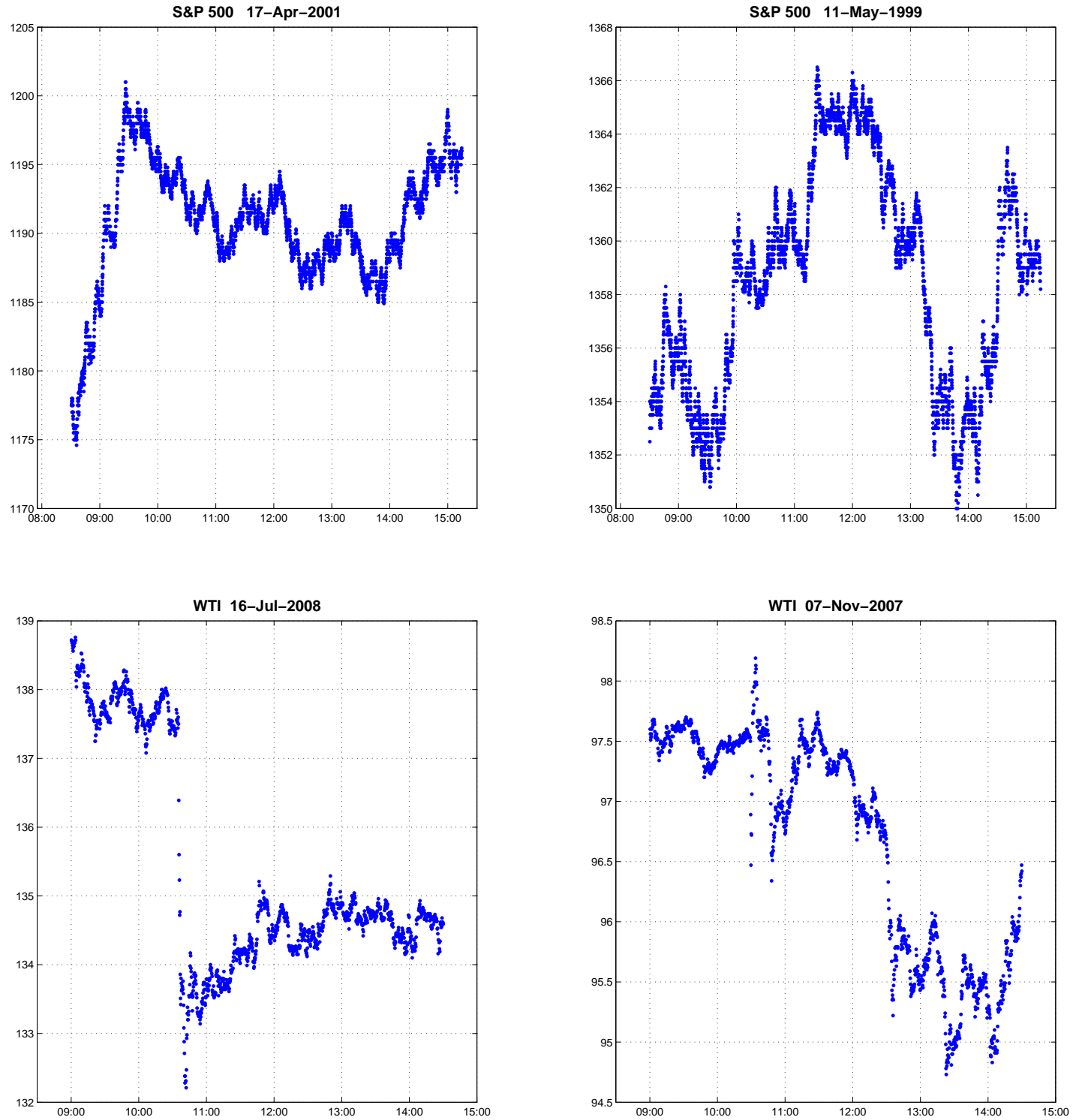
Figure 8: Jumpy days
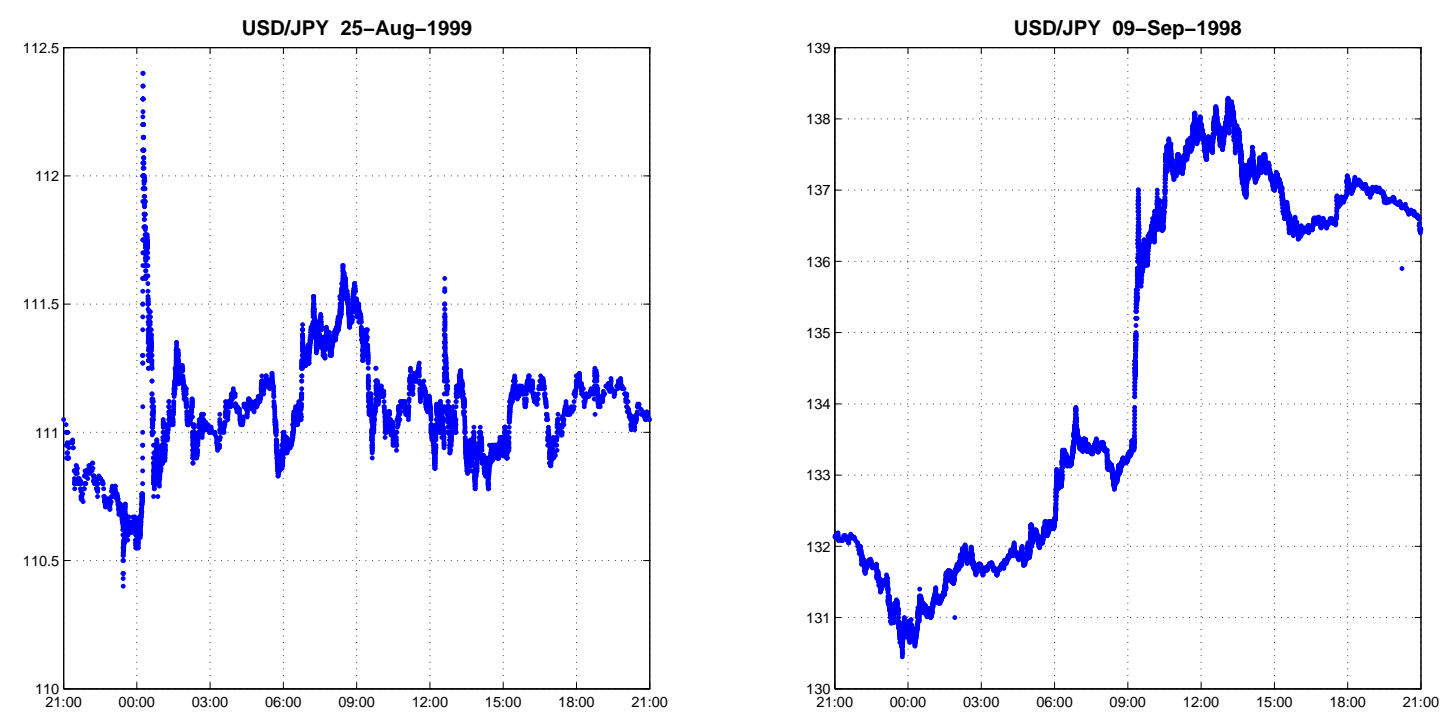
Figure 9: Average predictive likelihood based on Amisano and Giacomini (2007) for the density of S\&P 500 futures

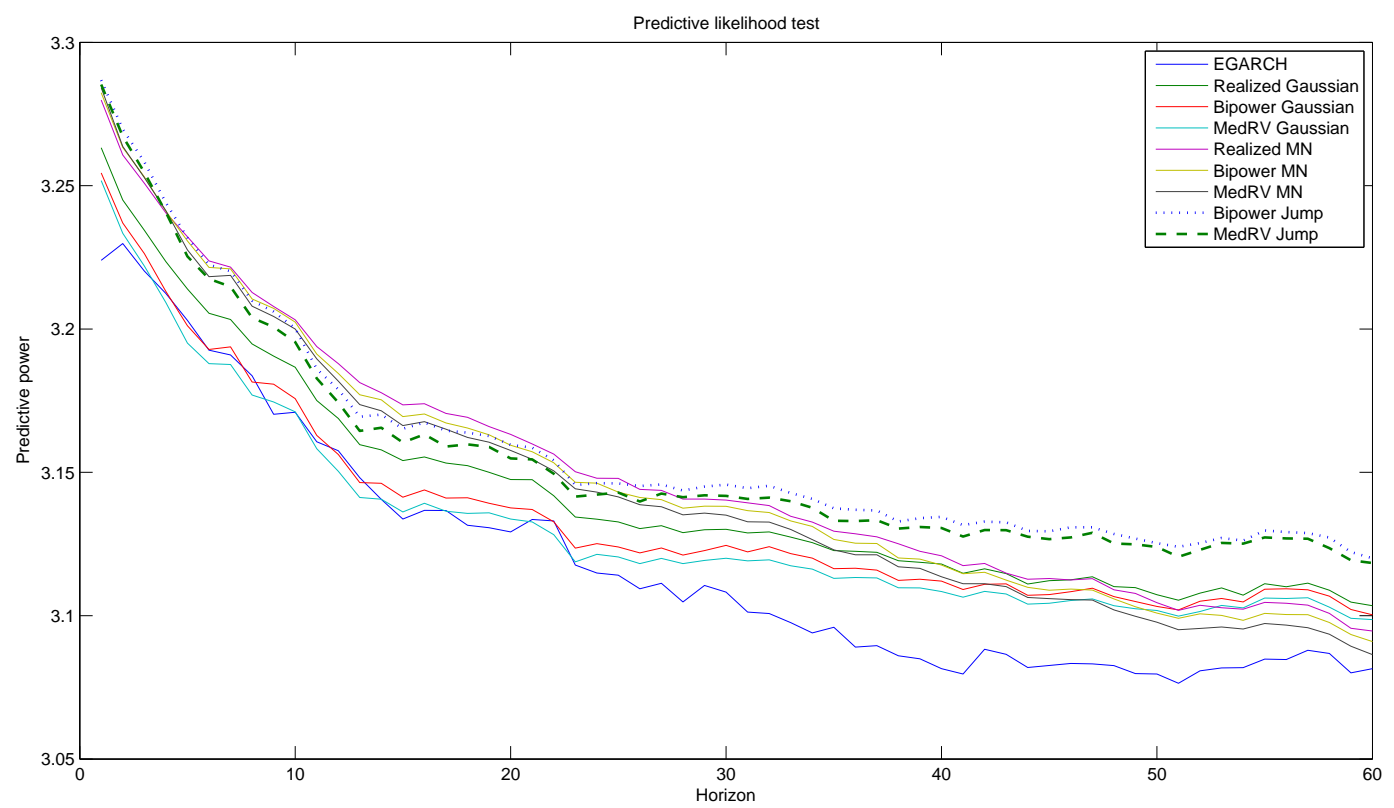

Figure 10: Average predictive likelihood based on Amisano and Giacomini (2007) for the density of WTI futures

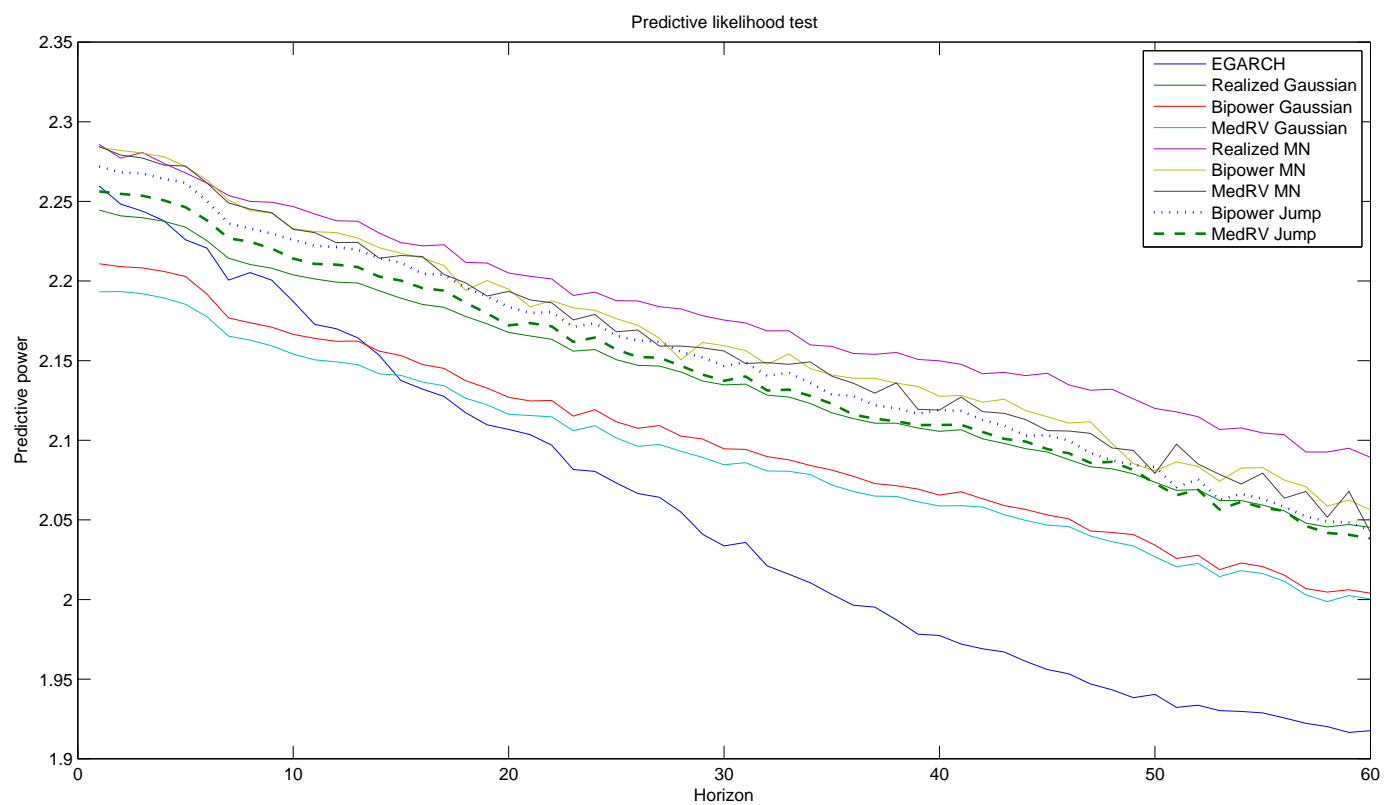


Figure 11: Average predictive likelihood based on Amisano and Giacomini (2007) for the density of USD/JPY

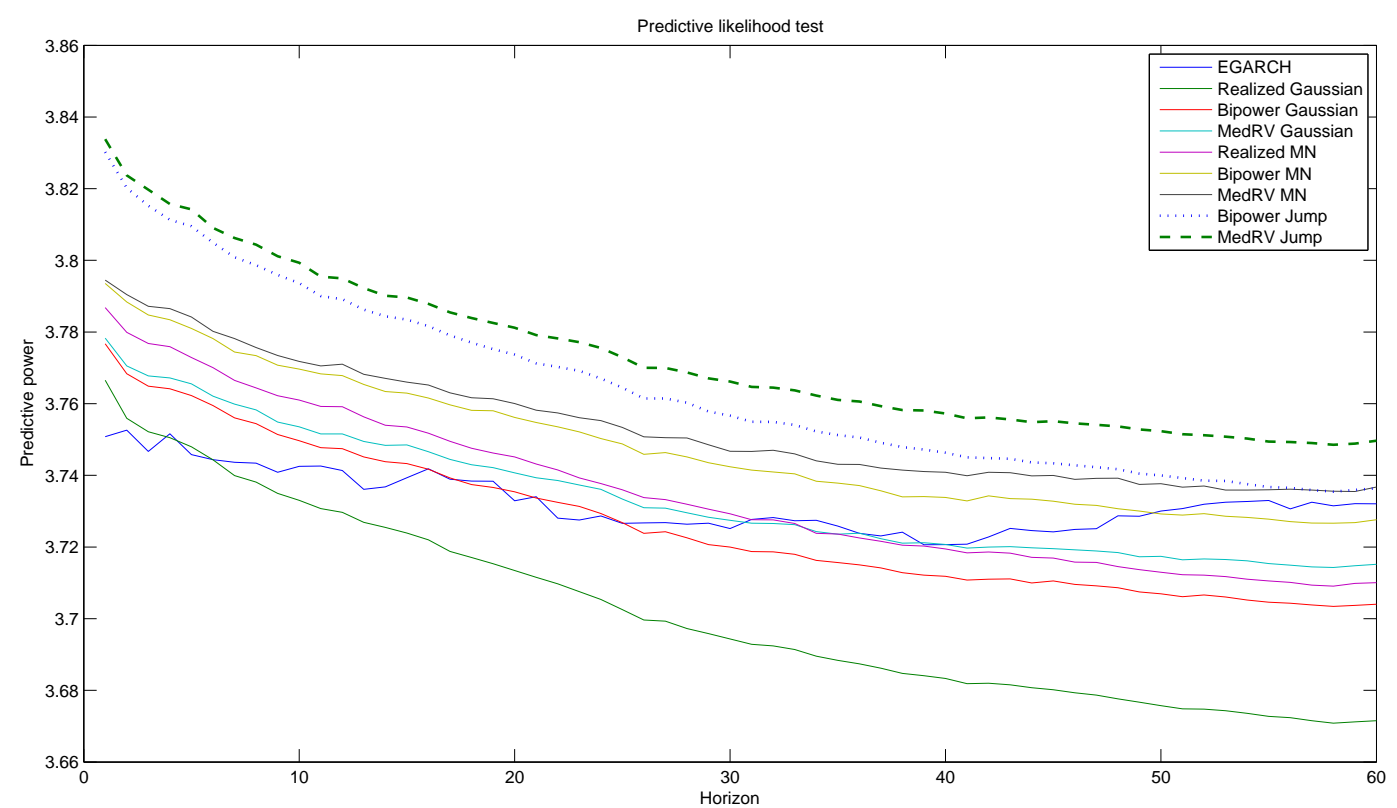




\section{Tables}

Table 1: Summary Statistics

\begin{tabular}{cccccccc}
\hline S\&P500 & $R_{t}$ & $R V_{t}$ & $B P V_{t}$ & $M e d R V_{t}$ & $\log \left(R V_{t}\right)$ & $\log \left(B P V_{t}\right)$ & $\log \left(M e d R V_{t}\right)$ \\
\hline Mean & 0.0515 & 0.1601 & 0.1559 & 0.1544 & -9.6046 & -9.6671 & -9.694 \\
Std. Dev. & 0.183 & 0.0021 & 0.0019 & 0.0019 & 0.8759 & 0.892 & 0.9012 \\
Skewness & -0.1334 & 6.8497 & 5.2274 & 5.3659 & 0.2984 & 0.2674 & 0.255 \\
Kurtosis & 3.2819 & 87.9456 & 44.1957 & 45.6785 & 0.0514 & -0.0452 & -0.0485 \\
& & & & & & & \\
Observations & 3135 & & & & & & \\
\hline
\end{tabular}

\begin{tabular}{cccccccc}
\hline WTI & $R_{t}$ & $R V_{t}$ & $B P V_{t}$ & $M e d R V_{t}$ & $\log \left(R V_{t}\right)$ & $\log \left(B P V_{t}\right)$ & $\log \left(M e d R V_{t}\right)$ \\
\hline Mean & 0.1445 & 0.3061 & 0.2954 & 0.2896 & -8.1738 & -8.2469 & -8.2860 \\
Std. Dev. & 0.3846 & 0.0061 & 0.0057 & 0.0055 & 0.6940 & 0.6971 & 0.6975 \\
Skewness & -0.1724 & 3.8354 & 3.9463 & 3.8867 & 0.6875 & 0.6670 & 0.6506 \\
Kurtosis & 3.2218 & 19.9500 & 22.0793 & 20.8598 & 0.7653 & 0.7854 & 0.7764 \\
& & & & & & & \\
Observations & 2058 & & & & & &
\end{tabular}

\begin{tabular}{cccccccc}
\hline USD/JPY & $R_{t}$ & $R V_{t}$ & $B P V_{t}$ & $M e d R V_{t}$ & $\log \left(R V_{t}\right)$ & $\log \left(B P V_{t}\right)$ & $\log \left(M e d R V_{t}\right)$ \\
\hline Mean & 0.0039 & 0.1155 & 0.1068 & 0.1044 & -10.1308 & -10.2755 & -10.3121 \\
Std. Dev. & 0.1087 & 0.0014 & 0.0011 & 0.0011 & 0.6756 & 0.6706 & 0.6561 \\
Skewness & -0.8422 & 22.2219 & 23.9665 & 24.9671 & 0.7511 & 0.6286 & 0.6807 \\
Kurtosis & 7.343 & 770.7384 & 872.5957 & 924.6845 & 1.9751 & 1.95 & 1.9959 \\
& & & & & & & \\
Observations & 2703 & & & & & & \\
\hline
\end{tabular}

Note: Mean values are given as the mean of the annualized squared root values. 
Table 2: Relative contribution of jumps in percents computed as the ratio between the difference between RV and BPV, when significant at a given threshold, and RV.

\begin{tabular}{cccc}
\hline Contribution of jumps & S\&P 500 & WTI crude oil & USD/JPY \\
\hline $0.1 \%$ threshold & $2.65 \%$ & $2.61 \%$ & $7.79 \%$ \\
$0.5 \%$ threshold & $3.21 \%$ & $3.37 \%$ & $9.22 \%$ \\
$1 \%$ threshold & $3.56 \%$ & $3.81 \%$ & $9.86 \%$ \\
$5 \%$ threshold & $4.53 \%$ & $5.06 \%$ & $11.88 \%$ \\
no threshold & $5.24 \%$ & $6.88 \%$ & $14.45 \%$ \\
\hline
\end{tabular}


Table 3: Kolmogorov-Smirnov statistics for daily open-to-close standardized returns. The distributions considered are the Gaussian, the symmetric Student- $t$, the Generalized Hyperbolic $(\mathrm{GH})$, the Normal Inverse Gaussian (NIG), the Hyperbolic (H) and the Mixture of Normals $(\mathrm{MN})$. The values in the table are $p$-values.

\begin{tabular}{cccc|ccc}
\hline & \multicolumn{3}{c}{ S\&P500 } & \multicolumn{4}{c}{ WTI } \\
\hline & $R V_{t}$ & $B P V_{t}$ & $M e d R V_{t}$ & $R V_{t}$ & $B P V_{t}$ & $M e d R V_{t}$ \\
\hline Gaussian & 0 & 0 & 0 & 0 & 0 & 0 \\
Student- $t$ & 0 & 0 & 0 & 0 & 0 & 0 \\
Generalized Hyperbolic & 0.93 & 0.42 & 0.15 & 0.94 & 0.55 & 0.95 \\
Normal Inverse Gaussian & 0.67 & 0.51 & 0.15 & 0.69 & 0.75 & 0.95 \\
Hyperbolic & 0.97 & 0.5 & 0.07 & 0.26 & 0.95 & 0.44 \\
Mixture of Gaussian & 0.25 & 0.45 & 0.58 & 0.84 & 0.95 & 0.96 \\
\hline
\end{tabular}

\begin{tabular}{cccc}
\hline & \multicolumn{3}{c}{ USD/JPY } \\
\hline & $R V_{t}$ & $B P V_{t}$ & $M e d R V_{t}$ \\
\hline Gaussian & 0.01 & 0.38 & 0.36 \\
Student- $t$ & 0 & 0.21 & 0.67 \\
Generalized Hyperbolic & 0.65 & 0.28 & 0.61 \\
Normal Inverse Gaussian & 0.28 & 0.63 & 0.88 \\
Hyperbolic & $\mathrm{NA}$ & $\mathrm{NA}$ & $\mathrm{NA}$ \\
Mixture of Gaussian & 0.21 & 0.53 & 0.67 \\
\hline
\end{tabular}


Table 4: Descriptive statistics about jumps extracted using the sequential procedure in Andersen et al. (2010). The chosen significance threshold is $1 \%$.

\begin{tabular}{cccc}
\hline Jump descriptive statistics & S\&P 500 & WTI crude oil & USD/JPY \\
\hline Total number of jumps & 433 & 329 & 308 \\
Number of days with jump(s) & 297 & 232 & 183 \\
Percentage of days with jump(s) & $9.47 \%$ & $11.15 \%$ & $6.78 \%$ \\
Average duration between two jumps (days) & 7.24 & 6.33 & 8.76 \\
Average duration between two days with jump(s) & 10.56 & 8.97 & 14.76 \\
Number of days with exactly 1 jump & 215 & 165 & 102 \\
Percentage of days with exactly 1 jump & $6.86 \%$ & $7.93 \%$ & $3.78 \%$ \\
Number of days with exactly 2 jump & 50 & 48 & 49 \\
Percentage of days with exactly 2 jump & $1.59 \%$ & $2.31 \%$ & $1.81 \%$ \\
Number of days with exactly 3 jump & 18 & 10 & 20 \\
Percentage of days with exactly 3 jump & $0.57 \%$ & $0.48 \%$ & $0.74 \%$ \\
Number of days with exactly 4 jump & 6 & 7 & 12 \\
Percentage of days with exactly 4 jump & $0.19 \%$ & $0.34 \%$ & $0.44 \%$ \\
Number of days with exactly 5 jump & 8 & 2 & - \\
Percentage of days with exactly 5 jump & $0.26 \%$ & $0.09 \%$ & $0 \%$ \\
\hline
\end{tabular}


Table 5: Estimation results for S\&P500 Futures.

\begin{tabular}{ccccc}
\hline EGARCH & $\omega$ & $\alpha$ & $\theta$ & $\beta$ \\
\hline Average & -0.328 & -0.129 & 0.078 & 0.970 \\
Standard Dev. & 0.196 & 0.038 & 0.027 & 0.021 \\
Skewness & -0.554 & -0.415 & -0.097 & -0.697 \\
Kurtosis & -0.953 & -1.104 & 0.372 & -0.817 \\
$\mathbf{5 \%}$ quantile & -0.681 & -0.190 & 0.033 & 0.931 \\
$\mathbf{9 5 \%}$ quantile & -0.087 & -0.079 & 0.123 & 0.993 \\
\hline
\end{tabular}

\begin{tabular}{cccccccc}
\hline HAR-RV-Gaussian & $\omega$ & $\phi_{1}$ & $\phi_{2}$ & $\phi_{3}$ & $\gamma$ & $\mu$ & $\eta$ \\
\hline Average & -0.956 & 0.283 & 0.432 & 0.185 & -0.105 & 0.000 & 0.445 \\
Standard Dev. & 0.429 & 0.045 & 0.072 & 0.023 & 0.013 & 0.000 & 0.037 \\
Skewness & -0.260 & -0.484 & 0.374 & -0.501 & -0.463 & 0.479 & 0.611 \\
Kurtosis & -1.327 & -1.170 & -1.320 & -0.846 & -1.022 & -1.191 & -0.698 \\
5\% quantile & -1.674 & 0.210 & 0.341 & 0.142 & -0.128 & 0.000 & 0.397 \\
95\% quantile & -0.409 & 0.341 & 0.550 & 0.216 & -0.088 & 0.000 & 0.520 \\
\hline
\end{tabular}

\begin{tabular}{cccccccc}
\hline HAR-BPV-Gaussian & $\omega$ & $\phi_{1}$ & $\phi_{2}$ & $\phi_{3}$ & $\gamma$ & $\mu$ & $\eta$ \\
\hline Average & -0.886 & 0.294 & 0.436 & 0.178 & -0.101 & 0.000 & 0.441 \\
Standard Dev. & 0.368 & 0.043 & 0.072 & 0.018 & 0.014 & 0.000 & 0.033 \\
Skewness & -0.229 & -0.404 & 0.232 & -0.372 & -0.511 & 0.479 & 0.836 \\
Kurtosis & -1.337 & -1.256 & -1.383 & -0.577 & -1.031 & -1.191 & -0.328 \\
5\% quantile & -1.500 & 0.227 & 0.342 & 0.147 & -0.126 & 0.000 & 0.402 \\
95\% quantile & -0.420 & 0.349 & 0.546 & 0.202 & -0.084 & 0.000 & 0.512 \\
\hline
\end{tabular}

\begin{tabular}{cccccccc}
\hline HAR-MedRV-Gaussian & $\omega$ & $\phi_{1}$ & $\phi_{2}$ & $\phi_{3}$ & $\gamma$ & $\mu$ & $\eta$ \\
\hline Average & -0.894 & 0.297 & 0.428 & 0.182 & -0.099 & 0.000 & 0.450 \\
Standard Dev. & 0.356 & 0.040 & 0.076 & 0.017 & 0.013 & 0.000 & 0.031 \\
Skewness & -0.200 & -0.447 & 0.209 & 0.207 & -0.381 & 0.479 & 0.848 \\
Kurtosis & -1.366 & -1.085 & -1.301 & -0.262 & -1.197 & -1.191 & -0.302 \\
5\% quantile & -1.484 & 0.227 & 0.324 & 0.156 & -0.121 & 0.000 & 0.414 \\
95\% quantile & -0.443 & 0.349 & 0.546 & 0.208 & -0.082 & 0.000 & 0.518 \\
\hline
\end{tabular}


Table 5 (continued): Estimation results for S\&P500 Futures.

\begin{tabular}{ccccccccccccc}
\hline HAR-RV-Mixture & $\omega$ & $\phi_{1}$ & $\phi_{2}$ & $\phi_{3}$ & $\gamma$ & $\phi$ & $\mu_{1}$ & $\sigma_{1}$ & $\mu_{2}$ & $\sigma_{2}$ & $\mu$ & $\eta$ \\
\hline Average & -0.945 & 0.283 & 0.433 & 0.188 & -0.104 & 0.267 & -1.071 & 1.134 & 0.302 & 1.098 & 0.000 & 0.445 \\
Standard Dev. & 0.436 & 0.042 & 0.071 & 0.022 & 0.011 & 0.187 & 0.586 & 0.215 & 0.164 & 0.204 & 0.000 & 0.037 \\
Skewness & -0.288 & -0.516 & 0.423 & -0.313 & -0.293 & 0.601 & -1.289 & 4.262 & 0.541 & 1.204 & 0.479 & 0.612 \\
Kurtosis & -1.319 & -1.028 & -1.254 & -0.940 & -1.117 & -0.828 & 2.971 & 21.666 & -0.609 & 0.249 & -1.191 & -0.697 \\
5\% quantile & -1.673 & 0.211 & 0.345 & 0.149 & -0.122 & 0.052 & -1.917 & 0.999 & 0.104 & 0.911 & 0.000 & 0.397 \\
95\% quantile & -0.381 & 0.338 & 0.553 & 0.221 & -0.087 & 0.623 & -0.380 & 1.368 & 0.625 & 1.554 & 0.000 & 0.520 \\
\hline
\end{tabular}

\begin{tabular}{ccccccccccccc}
\hline HAR-BPV-Mixture & $\omega$ & $\phi_{1}$ & $\phi_{2}$ & $\phi_{3}$ & $\gamma$ & $\phi$ & $\mu_{1}$ & $\sigma_{1}$ & $\mu_{2}$ & $\sigma_{2}$ & $\mu$ & $\eta$ \\
\hline Average & -0.889 & 0.296 & 0.436 & 0.178 & -0.099 & 0.235 & -1.143 & 1.073 & 0.318 & 1.007 & 0.000 & 0.441 \\
Standard Dev. & 0.368 & 0.040 & 0.070 & 0.017 & 0.012 & 0.125 & 0.343 & 0.096 & 0.125 & 0.119 & 0.000 & 0.033 \\
Skewness & -0.262 & -0.449 & 0.270 & -0.411 & -0.388 & 0.886 & -0.087 & 0.791 & 0.844 & 1.398 & 0.479 & 0.838 \\
Kurtosis & -1.327 & -1.100 & -1.325 & -0.569 & -1.125 & -0.226 & -0.681 & 0.247 & -0.210 & 0.906 & -1.191 & -0.326 \\
5\% quantile & -1.503 & 0.228 & 0.344 & 0.150 & -0.119 & 0.094 & -1.662 & 0.957 & 0.173 & 0.899 & 0.000 & 0.402 \\
95\% quantile & -0.429 & 0.350 & 0.547 & 0.202 & -0.083 & 0.500 & -0.582 & 1.273 & 0.583 & 1.289 & 0.000 & 0.512 \\
\hline
\end{tabular}

\begin{tabular}{|c|c|c|c|c|c|c|c|c|c|c|c|c|}
\hline HAR-MedRV-Mixture & $\omega$ & $\phi_{1}$ & $\phi_{2}$ & $\phi_{3}$ & $\gamma$ & $\phi$ & $\mu_{1}$ & $\sigma_{1}$ & $\mu_{2}$ & $\sigma_{2}$ & $\mu$ & $\eta$ \\
\hline Average & -0.902 & 0.301 & 0.428 & 0.181 & -0.096 & 0.236 & -1.172 & 1.068 & 0.333 & 0.989 & 0.000 & 0.450 \\
\hline Standard Dev. & 0.353 & 0.039 & 0.074 & 0.015 & 0.012 & 0.114 & 0.319 & 0.084 & 0.119 & 0.106 & 0.000 & 0.031 \\
\hline Skewness & -0.243 & -0.557 & 0.254 & 0.093 & -0.261 & 0.858 & -0.086 & 0.840 & 0.804 & 1.309 & 0.479 & 0.850 \\
\hline Kurtosis & -1.351 & -0.847 & -1.243 & -0.424 & -1.272 & -0.297 & -0.558 & 0.069 & -0.294 & 0.479 & -1.191 & -0.300 \\
\hline $5 \%$ quantile & -1.489 & 0.228 & 0.327 & 0.158 & -0.114 & 0.102 & -1.655 & 0.973 & 0.188 & 0.890 & 0.000 & 0.414 \\
\hline $95 \%$ quantile & -0.463 & 0.351 & 0.546 & 0.206 & -0.081 & 0.469 & -0.653 & 1.244 & 0.575 & 1.228 & 0.000 & 0.518 \\
\hline
\end{tabular}

\begin{tabular}{ccccccccccc}
\hline HAR-BPV-Jump & $\omega$ & $\phi_{1}$ & $\phi_{2}$ & $\phi_{3}$ & $\gamma$ & $\mu$ & $\eta$ & $\lambda$ & $\mu_{z}$ & $\sigma_{z}$ \\
\hline Average & -0.886 & 0.291 & 0.440 & 0.177 & -0.101 & 0.000 & 0.441 & 0.138 & 0.000 & 0.006 \\
Standard Dev. & 0.376 & 0.042 & 0.072 & 0.019 & 0.013 & 0.000 & 0.033 & 0.035 & 0.000 & 0.002 \\
Skewness & -0.259 & -0.410 & 0.209 & -0.190 & -0.376 & 0.514 & 0.848 & 0.484 & 0.399 & -0.229 \\
Kurtosis & -1.284 & -1.180 & -1.407 & -0.685 & -1.027 & -1.122 & -0.319 & -1.363 & 0.308 & -1.534 \\
5\% quantile & -1.524 & 0.224 & 0.343 & 0.144 & -0.123 & 0.000 & 0.402 & 0.102 & 0.000 & 0.003 \\
95\% quantile & -0.402 & 0.347 & 0.551 & 0.204 & -0.083 & 0.000 & 0.512 & 0.196 & 0.001 & 0.008 \\
\hline & & & & & & & & & & \\
\hline HAR-MedRV-Jump & $\omega$ & & & & & & & & & \\
Average & -0.892 & 0.296 & 0.431 & 0.181 & -0.099 & 0.000 & 0.450 & 0.138 & 0.000 & 0.006 \\
Standard Dev. & 0.362 & 0.040 & 0.077 & 0.019 & 0.013 & 0.000 & 0.031 & 0.035 & 0.000 & 0.002 \\
Skewness & -0.238 & -0.443 & 0.173 & 0.224 & -0.251 & 0.514 & 0.863 & 0.484 & 0.399 & -0.229 \\
Kurtosis & -1.306 & -1.034 & -1.338 & 0.012 & -1.207 & -1.122 & -0.287 & -1.363 & 0.308 & -1.534 \\
5\% quantile & -1.504 & 0.226 & 0.325 & 0.150 & -0.119 & 0.000 & 0.414 & 0.102 & 0.000 & 0.003 \\
95\% quantile & -0.428 & 0.348 & 0.549 & 0.209 & -0.081 & 0.000 & 0.518 & 0.196 & 0.001 & 0.008 \\
\hline
\end{tabular}


Table 6: Estimation results for WTI Futures.

\begin{tabular}{ccccc}
\hline EGARCH & $\omega$ & $\alpha$ & $\theta$ & $\beta$ \\
\hline Average & -0.563 & -0.078 & 0.104 & 0.938 \\
Standard Dev. & 0.404 & 0.029 & 0.014 & 0.052 \\
Skewness & -0.433 & -0.723 & -0.525 & -0.458 \\
Kurtosis & -1.014 & -0.292 & 0.728 & -0.970 \\
$\mathbf{5 \%}$ quantile & -1.240 & -0.128 & 0.075 & 0.849 \\
$\mathbf{9 5 \%}$ quantile & -0.149 & -0.047 & 0.123 & 0.990 \\
\hline
\end{tabular}

\begin{tabular}{cccccccc}
\hline HAR-RV-Gaussian & $\omega$ & $\phi_{1}$ & $\phi_{2}$ & $\phi_{3}$ & $\gamma$ & $\mu$ & $\eta$ \\
\hline Average & -0.736 & 0.076 & 0.543 & 0.294 & -0.041 & 0.001 & 0.422 \\
Standard Dev. & 0.444 & 0.007 & 0.033 & 0.026 & 0.003 & 0.000 & 0.004 \\
Skewness & -0.072 & 0.104 & -0.299 & -0.267 & -0.225 & 0.260 & -0.257 \\
Kurtosis & -1.842 & 0.484 & -1.487 & -0.954 & 0.138 & -1.016 & -0.898 \\
5\% quantile & -1.324 & 0.064 & 0.490 & 0.250 & -0.047 & 0.000 & 0.414 \\
95\% quantile & -0.255 & 0.086 & 0.582 & 0.331 & -0.036 & 0.001 & 0.428 \\
\hline
\end{tabular}

\begin{tabular}{cccccccc}
\hline HAR-BPV-Gaussian & $\omega$ & $\phi_{1}$ & $\phi_{2}$ & $\phi_{3}$ & $\gamma$ & $\mu$ & $\eta$ \\
\hline Average & -0.698 & 0.085 & 0.543 & 0.289 & -0.038 & 0.001 & 0.410 \\
Standard Dev. & 0.409 & 0.011 & 0.051 & 0.017 & 0.004 & 0.000 & 0.003 \\
Skewness & -0.080 & -0.121 & -0.089 & -0.883 & -0.259 & 0.260 & -0.262 \\
Kurtosis & -1.850 & -0.099 & -1.784 & 1.708 & -0.204 & -1.016 & -0.106 \\
5\% quantile & -1.232 & 0.066 & 0.476 & 0.258 & -0.045 & 0.000 & 0.405 \\
95\% quantile & -0.258 & 0.101 & 0.605 & 0.314 & -0.031 & 0.001 & 0.414 \\
\hline
\end{tabular}

\begin{tabular}{cccccccc}
\hline HAR-MedRV-Gaussian & $\omega$ & $\phi_{1}$ & $\phi_{2}$ & $\phi_{3}$ & $\gamma$ & $\mu$ & $\eta$ \\
\hline Average & -0.710 & 0.089 & 0.524 & 0.304 & -0.036 & 0.001 & 0.413 \\
Standard Dev. & 0.419 & 0.020 & 0.061 & 0.018 & 0.004 & 0.000 & 0.003 \\
Skewness & -0.107 & 0.152 & -0.123 & -0.347 & -0.124 & 0.260 & -0.014 \\
Kurtosis & -1.818 & -1.498 & -1.813 & -0.031 & -0.365 & -1.016 & -0.889 \\
5\% quantile & -1.277 & 0.061 & 0.442 & 0.275 & -0.042 & 0.000 & 0.409 \\
95\% quantile & -0.262 & 0.117 & 0.595 & 0.331 & -0.029 & 0.001 & 0.417 \\
\hline
\end{tabular}


Table 6 (continued): Estimation results for WTI Futures.

\begin{tabular}{ccccccccccccc}
\hline HAR-RV-Mixture & $\omega$ & $\phi_{1}$ & $\phi_{2}$ & $\phi_{3}$ & $\gamma$ & $\phi$ & $\mu_{1}$ & $\sigma_{1}$ & $\mu_{2}$ & $\sigma_{2}$ & $\mu$ & $\eta$ \\
\hline Average & -0.693 & 0.100 & 0.524 & 0.298 & -0.038 & 0.449 & -0.763 & 1.057 & 0.626 & 1.238 & 0.001 & 0.422 \\
Standard Dev. & 0.395 & 0.027 & 0.068 & 0.049 & 0.005 & 0.173 & 0.267 & 0.119 & 0.259 & 0.217 & 0.000 & 0.004 \\
Skewness & -0.232 & -0.112 & -0.493 & -0.215 & -0.004 & -0.654 & -1.672 & 5.545 & -0.272 & 0.828 & 0.260 & -0.242 \\
Kurtosis & -1.403 & 2.986 & 1.660 & 3.405 & 3.367 & -0.245 & 3.595 & 32.467 & -0.434 & 1.303 & -1.016 & -0.851 \\
5\% quantile & -1.282 & 0.060 & 0.413 & 0.223 & -0.046 & 0.098 & -1.323 & 1.000 & 0.160 & 0.944 & 0.000 & 0.414 \\
95\% quantile & -0.201 & 0.140 & 0.616 & 0.373 & -0.030 & 0.677 & -0.471 & 1.118 & 1.011 & 1.623 & 0.001 & 0.428 \\
\hline
\end{tabular}

\begin{tabular}{ccccccccccccc}
\hline HAR-BPV-Mixture & $\omega$ & $\phi_{1}$ & $\phi_{2}$ & $\phi_{3}$ & $\gamma$ & $\phi$ & $\mu_{1}$ & $\sigma_{1}$ & $\mu_{2}$ & $\sigma_{2}$ & $\mu$ & $\eta$ \\
\hline Average & -0.695 & 0.119 & 0.540 & 0.263 & -0.032 & 0.486 & -0.757 & 1.084 & 0.762 & 1.325 & 0.001 & 0.410 \\
Standard Dev. & 0.411 & 0.041 & 0.068 & 0.054 & 0.009 & 0.219 & 0.324 & 0.178 & 0.379 & 0.350 & 0.000 & 0.003 \\
Skewness & -0.319 & 0.464 & -0.474 & -0.407 & -0.182 & -0.636 & -1.446 & 3.648 & -0.239 & 1.629 & 0.260 & -0.041 \\
Kurtosis & -0.898 & 2.343 & 1.962 & 2.900 & 8.250 & -0.586 & 2.359 & 12.512 & -0.572 & 6.993 & -1.016 & 0.471 \\
5\% quantile & -1.348 & 0.060 & 0.424 & 0.175 & -0.044 & 0.046 & -1.462 & 1.001 & 0.050 & 0.914 & 0.000 & 0.405 \\
95\% quantile & -0.144 & 0.190 & 0.656 & 0.344 & -0.020 & 0.769 & -0.385 & 1.576 & 1.316 & 1.949 & 0.001 & 0.415 \\
\hline
\end{tabular}

\begin{tabular}{|c|c|c|c|c|c|c|c|c|c|c|c|c|}
\hline HAR-MedRV-Mixture & $\omega$ & $\phi_{1}$ & $\phi_{2}$ & $\phi_{3}$ & $\bar{\gamma}$ & $\phi$ & $\overline{\mu_{1}}$ & $\overline{\sigma_{1}}$ & $\overline{\mu_{2}}$ & $\overline{\sigma_{2}}$ & $\mu$ & $\eta$ \\
\hline Average & -0.666 & 0.110 & 0.543 & 0.273 & -0.030 & 0.518 & -0.748 & 1.054 & 0.832 & 1.300 & 0.001 & 0.413 \\
\hline Standard Dev. & 0.383 & 0.036 & 0.062 & 0.041 & 0.007 & 0.164 & 0.239 & 0.100 & 0.299 & 0.274 & 0.000 & 0.003 \\
\hline Skewness & -0.261 & 0.261 & -0.610 & 0.584 & -0.709 & -0.841 & -1.572 & 5.561 & -0.375 & 0.972 & 0.260 & -0.049 \\
\hline Kurtosis & -0.989 & 2.461 & 3.723 & 1.194 & 6.061 & 0.689 & 4.999 & 31.912 & 0.094 & 0.898 & -1.016 & -0.854 \\
\hline $5 \%$ quantile & -1.247 & 0.051 & 0.447 & 0.215 & -0.040 & 0.186 & -1.162 & 1.007 & 0.280 & 0.952 & 0.000 & 0.409 \\
\hline $95 \%$ quantile & -0.161 & 0.167 & 0.645 & 0.346 & -0.021 & 0.743 & -0.427 & 1.066 & 1.274 & 1.820 & 0.001 & 0.417 \\
\hline
\end{tabular}

\begin{tabular}{ccccccccccc}
\hline HAR-BPV-Jump & $\omega$ & $\phi_{1}$ & $\phi_{2}$ & $\phi_{3}$ & $\gamma$ & $\mu$ & $\eta$ & $\lambda$ & $\mu_{z}$ & $\sigma_{z}$ \\
\hline Average & -0.704 & 0.084 & 0.544 & 0.288 & -0.038 & 0.001 & 0.410 & 0.149 & -0.001 & 0.010 \\
Standard Dev. & 0.412 & 0.011 & 0.051 & 0.018 & 0.004 & 0.000 & 0.003 & 0.014 & 0.001 & 0.000 \\
Skewness & -0.106 & -0.072 & -0.015 & -0.549 & -0.256 & 0.260 & -0.260 & -0.308 & -0.199 & 0.479 \\
Kurtosis & -1.814 & 0.113 & -1.676 & 0.548 & -0.167 & -1.016 & -0.115 & -0.088 & -0.694 & -1.117 \\
5\% quantile & -1.273 & 0.065 & 0.478 & 0.254 & -0.044 & 0.000 & 0.405 & 0.121 & -0.003 & 0.010 \\
95\% quantile & -0.261 & 0.101 & 0.612 & 0.315 & -0.031 & 0.001 & 0.414 & 0.171 & 0.000 & 0.011 \\
\hline & & & & & & & & & & \\
\hline HAR-MedRV-Jump & $\omega$ & & & & & & & & & \\
Average & -0.719 & 0.088 & 0.523 & 0.303 & -0.036 & 0.001 & 0.413 & 0.156 & -0.002 & 0.011 \\
Standard Dev. & 0.419 & 0.020 & 0.060 & 0.018 & 0.004 & 0.000 & 0.003 & 0.015 & 0.001 & 0.000 \\
Skewness & -0.112 & 0.129 & -0.094 & -0.143 & -0.143 & 0.260 & -0.013 & -0.544 & 0.302 & 0.432 \\
Kurtosis & -1.806 & -1.473 & -1.779 & -0.264 & -0.274 & -1.016 & -0.874 & 0.057 & -0.827 & -1.277 \\
5\% quantile & -1.292 & 0.062 & 0.444 & 0.275 & -0.042 & 0.000 & 0.409 & 0.123 & -0.003 & 0.010 \\
95\% quantile & -0.265 & 0.116 & 0.596 & 0.333 & -0.029 & 0.001 & 0.417 & 0.177 & -0.001 & 0.012 \\
\hline
\end{tabular}


Table 7: Estimation results for USD/JPY.

\begin{tabular}{ccccc}
\hline EGARCH & $\omega$ & $\alpha$ & $\theta$ & $\beta$ \\
\hline Average & -0.734 & -0.047 & 0.103 & 0.934 \\
Standard Dev. & 0.583 & 0.060 & 0.060 & 0.056 \\
Skewness & -1.386 & -1.314 & 1.066 & -1.442 \\
Kurtosis & 0.729 & 0.173 & -0.669 & 0.905 \\
$\mathbf{5 \%}$ quantile & -2.098 & -0.183 & 0.056 & 0.800 \\
$\mathbf{9 5 \%}$ quantile & -0.160 & -0.002 & 0.214 & 0.988 \\
\hline
\end{tabular}

\begin{tabular}{cccccccc}
\hline HAR-RV-Gaussian & $\omega$ & $\phi_{1}$ & $\phi_{2}$ & $\phi_{3}$ & $\gamma$ & $\mu$ & $\eta$ \\
\hline Average & -1.943 & 0.251 & 0.286 & 0.277 & -0.050 & 0.000 & 0.449 \\
Standard Dev. & 0.343 & 0.030 & 0.050 & 0.055 & 0.046 & 0.000 & 0.021 \\
Skewness & -0.061 & -0.017 & 0.085 & 0.444 & -0.353 & -0.039 & 0.984 \\
Kurtosis & -0.856 & -1.393 & -1.355 & -0.154 & -1.625 & -0.278 & 0.090 \\
5\% quantile & -2.546 & 0.209 & 0.216 & 0.194 & -0.116 & 0.000 & 0.425 \\
95\% quantile & -1.395 & 0.294 & 0.363 & 0.391 & -0.003 & 0.000 & 0.493 \\
\hline
\end{tabular}

\begin{tabular}{cccccccc}
\hline HAR-BPV-Gaussian & $\omega$ & $\phi_{1}$ & $\phi_{2}$ & $\phi_{3}$ & $\gamma$ & $\mu$ & $\eta$ \\
\hline Average & -1.743 & 0.283 & 0.315 & 0.237 & -0.039 & 0.000 & 0.433 \\
Standard Dev. & 0.305 & 0.023 & 0.044 & 0.041 & 0.040 & 0.000 & 0.018 \\
Skewness & -0.221 & 0.105 & -0.081 & 0.684 & -0.344 & -0.039 & 1.154 \\
Kurtosis & -0.801 & -1.371 & -1.370 & 0.409 & -1.638 & -0.278 & 0.637 \\
5\% quantile & -2.318 & 0.249 & 0.249 & 0.178 & -0.098 & 0.000 & 0.413 \\
95\% quantile & -1.274 & 0.316 & 0.382 & 0.327 & 0.003 & 0.000 & 0.473 \\
\hline
\end{tabular}

\begin{tabular}{cccccccc}
\hline HAR-MedRV-Gaussian & $\omega$ & $\phi_{1}$ & $\phi_{2}$ & $\phi_{3}$ & $\gamma$ & $\mu$ & $\eta$ \\
\hline Average & -1.742 & 0.292 & 0.319 & 0.224 & -0.035 & 0.000 & 0.420 \\
Standard Dev. & 0.320 & 0.020 & 0.042 & 0.035 & 0.037 & 0.000 & 0.019 \\
Skewness & -0.105 & 0.088 & -0.194 & 1.017 & -0.358 & -0.039 & 1.170 \\
Kurtosis & -0.930 & -1.065 & -1.346 & 1.147 & -1.593 & -0.278 & 0.661 \\
5\% quantile & -2.306 & 0.259 & 0.252 & 0.175 & -0.091 & 0.000 & 0.399 \\
95\% quantile & -1.242 & 0.321 & 0.376 & 0.308 & 0.005 & 0.000 & 0.463 \\
\hline
\end{tabular}


Table 7 (continued): Estimation results for USD/JPY.

\begin{tabular}{ccccccccccccc}
\hline HAR-RV-Mixture & $\omega$ & $\phi_{1}$ & $\phi_{2}$ & $\phi_{3}$ & $\gamma$ & $\phi$ & $\mu_{1}$ & $\sigma_{1}$ & $\mu_{2}$ & $\sigma_{2}$ & $\mu$ & $\eta$ \\
\hline Average & -1.850 & 0.249 & 0.296 & 0.279 & -0.047 & 0.150 & -0.232 & 1.709 & 0.037 & 1.080 & 0.000 & 0.449 \\
Standard Dev. & 0.375 & 0.034 & 0.046 & 0.049 & 0.042 & 0.031 & 0.134 & 0.099 & 0.012 & 0.023 & 0.000 & 0.021 \\
Skewness & -0.009 & 0.095 & 0.240 & 0.596 & -0.321 & 0.601 & -1.678 & 1.121 & 0.985 & 0.365 & -0.039 & 0.984 \\
Kurtosis & -1.145 & -1.237 & -0.926 & 0.280 & -1.653 & 3.067 & 1.987 & 1.728 & 0.682 & 3.174 & -0.278 & 0.090 \\
5\% quantile & -2.479 & 0.200 & 0.231 & 0.204 & -0.107 & 0.095 & -0.537 & 1.592 & 0.025 & 1.037 & 0.000 & 0.425 \\
95\% quantile & -1.302 & 0.302 & 0.374 & 0.389 & -0.002 & 0.194 & -0.125 & 1.921 & 0.062 & 1.111 & 0.000 & 0.493 \\
\hline
\end{tabular}

\begin{tabular}{|c|c|c|c|c|c|c|c|c|c|c|c|c|}
\hline HAR-BPV-Mixture & $\omega$ & $\phi_{1}$ & $\phi_{2}$ & $\phi_{3}$ & $\gamma$ & $\phi$ & $\overline{\mu_{1}}$ & $\sigma_{1}$ & $\overline{\mu_{2}}$ & $\overline{\sigma_{2}}$ & $\mu$ & $\eta$ \\
\hline Average & -1.669 & 0.285 & 0.325 & 0.233 & -0.035 & 0.159 & -0.279 & 1.600 & 0.039 & 1.085 & 0.000 & 0.433 \\
\hline Standa & 0.354 & 0.025 & 0. & 0.036 & 0.037 & 0.050 & 0.256 & 0.117 & 0.017 & 41 & 0.000 & 0.018 \\
\hline Ske & 0.134 & 0.371 & 0. & 0.607 & -0.306 & 0.341 & -2.118 & 0.844 & & -0.001 & -0.039 & 1.155 \\
\hline Ku & .025 & -0 . & & & & & & & & & 78 & 637 \\
\hline $5 \%$ & 252 & & & & -0 . & 0 . & -0 & 1. & & 0 & 0.000 & 0.413 \\
\hline $95 \%$ quantile & -1.097 & 0.324 & 0.392 & 0.310 & 0.004 & 0.253 & -0.006 & 1.830 & 0.070 & 1.157 & 0.000 & 0.473 \\
\hline
\end{tabular}

\begin{tabular}{ccccccccccccc}
\hline HAR-MedRV-Mixture & $\omega$ & $\phi_{1}$ & $\phi_{2}$ & $\phi_{3}$ & $\gamma$ & $\phi$ & $\mu_{1}$ & $\sigma_{1}$ & $\mu_{2}$ & $\sigma_{2}$ & $\mu$ & $\eta$ \\
\hline Average & -1.671 & 0.295 & 0.328 & 0.220 & -0.031 & 0.158 & -0.311 & 1.586 & 0.042 & 1.083 & 0.000 & 0.420 \\
Standard Dev. & 0.350 & 0.024 & 0.042 & 0.036 & 0.034 & 0.051 & 0.321 & 0.121 & 0.017 & 0.043 & 0.000 & 0.019 \\
Skewness & 0.232 & 0.527 & 0.015 & 0.600 & -0.313 & -0.231 & -2.493 & 1.101 & 0.294 & -0.703 & -0.039 & 1.172 \\
Kurtosis & -0.981 & -0.001 & -0.764 & 0.813 & -1.628 & 0.200 & 7.053 & 0.956 & 1.056 & 1.009 & -0.278 & 0.663 \\
5\% quantile & -2.206 & 0.260 & 0.260 & 0.164 & -0.081 & 0.063 & -1.040 & 1.441 & 0.011 & 0.998 & 0.000 & 0.399 \\
95\% quantile & -1.091 & 0.335 & 0.395 & 0.301 & 0.006 & 0.241 & -0.035 & 1.829 & 0.074 & 1.148 & 0.000 & 0.463 \\
\hline
\end{tabular}

\begin{tabular}{ccccccccccc}
\hline HAR-BPV-Jump & $\omega$ & $\phi_{1}$ & $\phi_{2}$ & $\phi_{3}$ & $\gamma$ & $\mu$ & $\eta$ & $\lambda$ & $\mu_{z}$ & $\sigma_{z}$ \\
\hline Average & -1.791 & 0.286 & 0.328 & 0.218 & -0.030 & 0.000 & 0.434 & 1.190 & 0.000 & 0.002 \\
Standard Dev. & 0.321 & 0.024 & 0.038 & 0.037 & 0.035 & 0.000 & 0.019 & 0.062 & 0.000 & 0.000 \\
Skewness & -0.144 & 0.046 & -0.211 & 1.009 & -0.329 & 0.044 & 1.080 & 0.113 & 0.250 & 0.546 \\
Kurtosis & -0.939 & -1.278 & -1.179 & 0.988 & -1.624 & -0.194 & 0.433 & -0.961 & 0.562 & -1.363 \\
5\% quantile & -2.357 & 0.248 & 0.267 & 0.167 & -0.084 & 0.000 & 0.413 & 1.101 & 0.000 & 0.002 \\
95\% quantile & -1.307 & 0.321 & 0.380 & 0.307 & 0.007 & 0.000 & 0.475 & 1.296 & 0.000 & 0.003 \\
\hline
\end{tabular}

\begin{tabular}{ccccccccccc}
\hline HAR-MedRV-Jump & $\omega$ & $\phi_{1}$ & $\phi_{2}$ & $\phi_{3}$ & $\gamma$ & $\mu$ & $\eta$ & $\lambda$ & $\mu_{z}$ & $\sigma_{z}$ \\
Average & -1.797 & 0.297 & 0.332 & 0.202 & -0.027 & 0.000 & 0.421 & 1.190 & 0.000 & 0.002 \\
Standard Dev. & 0.353 & 0.021 & 0.038 & 0.033 & 0.033 & 0.000 & 0.020 & 0.062 & 0.000 & 0.000 \\
Skewness & -0.126 & -0.039 & -0.277 & 1.247 & -0.325 & 0.044 & 1.108 & 0.113 & 0.250 & 0.546 \\
Kurtosis & -1.043 & -1.009 & -1.110 & 1.578 & -1.586 & -0.194 & 0.485 & -0.961 & 0.562 & -1.363 \\
5\% quantile & -2.422 & 0.261 & 0.268 & 0.160 & -0.076 & 0.000 & 0.399 & 1.101 & 0.000 & 0.002 \\
95\% quantile & -1.280 & 0.329 & 0.385 & 0.285 & 0.008 & 0.000 & 0.465 & 1.296 & 0.000 & 0.003 \\
\hline
\end{tabular}


Table 8: Average Diebold-Mariano (1995) pairwise test statistic with 5-, 10-, 30-, and 60-day horizon for S\&P500 Futures.

\begin{tabular}{|c|c|c|c|c|c|c|c|c|c|}
\hline $\begin{array}{c}\text { 5-day horizon } \\
\text { EGARCH } \\
\text { Eealized Gaussian } \\
\text { Bipower Gaussian } \\
\text { MedRV Gaussian } \\
\text { Realized MN } \\
\text { Bipower MN } \\
\text { MedRV MN } \\
\text { Bipower Jump } \\
\text { MedRV Jump } \\
\end{array}$ & EGARCH & $\begin{array}{c}\text { Realized Gaussian } \\
-2.638\end{array}$ & $\begin{array}{c}\text { Bipower Gaussian } \\
0.291 \\
2.428\end{array}$ & $\begin{array}{c}\text { MedRV Gaussian } \\
1.260 \\
2.400 \\
2.230\end{array}$ & $\begin{array}{c}\text { Realized MN } \\
-3.533 \\
-2.098 \\
-2.291 \\
-2.320\end{array}$ & $\begin{array}{c}\text { Bipower MN } \\
-3.002 \\
-2.609 \\
-2.764 \\
-2.737 \\
0.420\end{array}$ & $\begin{array}{c}\text { MedRV MN } \\
-2.338 \\
-2.440 \\
-26.063 \\
-2.914 \\
0.792 \\
1.310\end{array}$ & $\begin{array}{c}\text { Bipower Jump } \\
-4.248 \\
-6.993 \\
-5.266 \\
-5.581 \\
0.086 \\
-0.236 \\
-0.933\end{array}$ & $\begin{array}{c}\text { MedRV Jump } \\
-3.054 \\
-4.469 \\
-3.731 \\
-5.086 \\
1.757 \\
1.115 \\
0.450 \\
2.177\end{array}$ \\
\hline $\begin{array}{c}\text { 10-day horizon } \\
\text { EGARCH } \\
\text { Realized Gausian } \\
\text { Bipower Gaussian } \\
\text { MedRV Gaussian } \\
\text { Realized MN } \\
\text { Bipower MN } \\
\text { MedRV MN } \\
\text { Bipower Jump } \\
\text { MedRV Jump } \\
\text { Medur Jump }\end{array}$ & EGARCH & $\begin{array}{c}\text { Realized Gaussian } \\
-3.050\end{array}$ & $\begin{array}{c}\text { Bipower Gaussian } \\
-0.566 \\
2.545\end{array}$ & $\begin{array}{c}\text { MedRV Gaussian } \\
-0.013 \\
3.263 \\
4.663\end{array}$ & $\begin{array}{c}\text { Realized MN } \\
-3.538 \\
-6.566 \\
-3.234 \\
-3.294\end{array}$ & $\begin{array}{c}\text { Bipower MN } \\
-3.810 \\
-2.282 \\
-4.052 \\
-3.951 \\
0.378\end{array}$ & $\begin{array}{c}\text { MedRV MN } \\
-3.312 \\
-1.784 \\
-4.813 \\
-4.184 \\
1.740 \\
1.961\end{array}$ & $\begin{array}{c}\text { Bipower Jump } \\
-3.211 \\
-2.496 \\
-4.550 \\
-5.198 \\
0.296 \\
0.260 \\
-0.040\end{array}$ & $\begin{array}{c}\text { MedRV Jump } \\
-2.425 \\
-1.298 \\
-12.159 \\
-4.384 \\
0.651 \\
0.681 \\
0.481 \\
14.063\end{array}$ \\
\hline $\begin{array}{l}\text { 30-day horizon } \\
\text { EGARCH } \\
\text { Realized Gaussian } \\
\text { Bipower Gaussian } \\
\text { MedRV Gaussian } \\
\text { Realized MN } \\
\text { Bipower MN } \\
\text { MedRV MN } \\
\text { Bipower Jump } \\
\text { MedRV Jump } \\
\end{array}$ & & $\begin{array}{c}\text { Realized Gaussian } \\
-2.308\end{array}$ & $\begin{array}{c}\text { Bipower Gaussian } \\
-1.182 \\
2.878\end{array}$ & $\begin{array}{c}\text { MedRV Gaussian } \\
-1.256 \\
4.669 \\
4.094\end{array}$ & $\begin{array}{c}\text { Realized MN } \\
-2.6619 \\
-1.748 \\
-2.003 \\
-2.554\end{array}$ & $\begin{array}{c}\text { Bipower MN } \\
-2.448 \\
-1.428 \\
-1.796 \\
-2.366 \\
1.458\end{array}$ & $\begin{array}{c}\text { MedRV MN } \\
-2.092 \\
-0.829 \\
-1.340 \\
-1.899 \\
2.857 \\
4.790\end{array}$ & $\begin{array}{c}\text { Bipower Jump } \\
-4.529 \\
-5.628 \\
-3.798 \\
-4.571 \\
-0.621 \\
-0.881 \\
-1.214\end{array}$ & $\begin{array}{c}\text { MedRV Jum } \\
-3.879 \\
-3.901 \\
-3.024 \\
-3.894 \\
-0.164 \\
-0.411 \\
-0.749 \\
3.554\end{array}$ \\
\hline
\end{tabular}

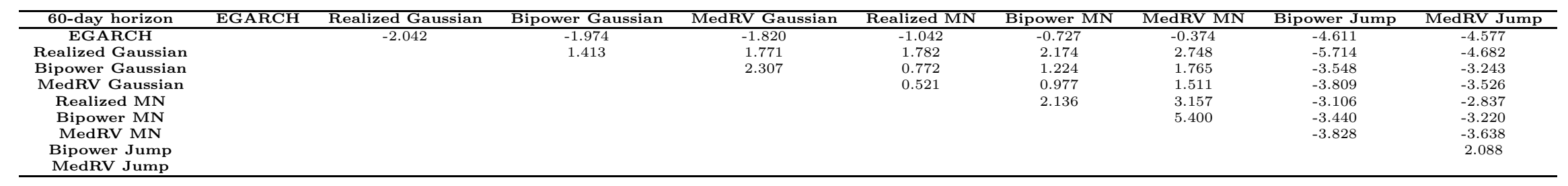
Note: this table presents the results of the average Diebold-Mariano (1995) pairwise test statistic with 5-. 10-. 30-. and 60-day horizon. The table reads the following way: a significant positive (negative)
estimated value rejects the null of equal performance between competing forecasts, and provides evidence in favor of model A (B). 
Table 9: Average Diebold-Mariano (1995) pairwise test statistic with 5-. 10-. 30-. and 60-day horizon for WTI Futures.

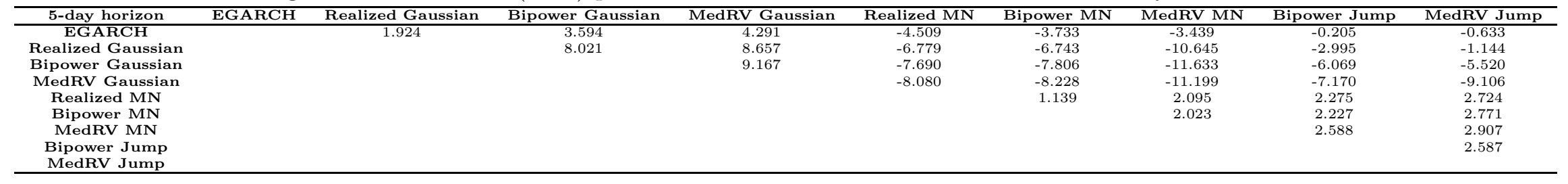

\begin{tabular}{|c|c|c|c|c|c|c|c|c|c|}
\hline 10-day horizon & $\overline{\text { EGARCH }}$ & Realized Gaussian & Bipower Gaussian & MedRV Gaussian & Realized MN & Bipower MN & MedRV MN & Bipower Jump & MedRV Jump \\
\hline & & & & & & & & & \\
\hline $\begin{array}{l}\text { Realizad Gaussia } \\
\text { a }\end{array}$ & & & $\begin{array}{l}1.0966 \\
10306\end{array}$ & $\begin{array}{l}6.005 \\
12469\end{array}$ & 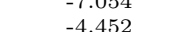 & $\begin{array}{l}-3.38 \\
-344\end{array}$ & $\begin{array}{l}-12.148 \\
-4.732\end{array}$ & $\begin{array}{l}-0.81 \\
-2.734\end{array}$ & 年 \\
\hline Bipower Gaussian & & & & 15.213 & -5.976 & -2.235 & -6.792 & -5.897 & $\begin{array}{l}-9.110 \\
-9.110\end{array}$ \\
\hline $\begin{array}{l}\text { MedRV Gaussian } \\
\text { Realized MN }\end{array}$ & & & & & & $\begin{array}{l}-5.982 \\
1.776\end{array}$ & $\begin{array}{l}-7.684 \\
4.001\end{array}$ & $\begin{array}{l}-7.009 \\
1.567\end{array}$ & $\begin{array}{l}-11.586 \\
2.217\end{array}$ \\
\hline Bipower MN & & & & & & & & 0.973 & 1.697 \\
\hline $\begin{array}{l}\text { MedRV MN } \\
\text { Bipower Jump }\end{array}$ & & & & & & & & & $\begin{array}{l}2.138 \\
2.400\end{array}$ \\
\hline MedRV Jump & & & & & & & & & \\
\hline
\end{tabular}

\begin{tabular}{|c|c|c|c|c|c|c|c|c|c|}
\hline 30-day horizon & EGARCH & Realized Gaussian & Bipower Gaussian & MedRV Gaussian & Realized MN & Bipower MN & MedRV MN & Bipower Jump & MedRV Jump \\
\hline EGARCH & & -3.874 & -2.211 & -1.154 & -3.355 & -3.561 & -2.915 & -6.019 & -4.824 \\
\hline Realized Gaussian & & & 4.717 & 4.515 & -2.381 & -2.691 & -1.459 & -4.998 & -0.912 \\
\hline Bipower Gaussian & & & & 4.139 & -2.934 & -3.254 & -2.418 & -5.760 & -7.116 \\
\hline MedRV Gaussian & & & & & -3.118 & -3.458 & -2.741 & -6.751 & -15.612 \\
\hline Realized MN & & & & & & 0.702 & 2.984 & 0.769 & 1.127 \\
\hline Bipower MN & & & & & & & 5.294 & 0.781 & 1.159 \\
\hline MedRV MN & & & & & & & & -0.121 & 0.457 \\
\hline Bipower Jump & & & & & & & & & 1.772 \\
\hline
\end{tabular}

\begin{tabular}{|c|c|c|c|c|c|c|c|c|c|}
\hline 60-day horizon & EGARCH & Realized Gaussian & Bipower Gaussian & MedRV Gaussian & Realized MN & Bipower MN & MedRV MN & Bipower Jump & $\frac{\text { MedRV Jump }}{2}$ \\
\hline EGARCH & & -3.572 & -2.229 & -1.526 & $\frac{-2.647}{-1}$ & $\frac{-3.139}{-139}>\mathrm{C}$ & -1.923 & -4.842 & -3.847 \\
\hline Realized Gaussian & & & 3.009 & 2.874 & -1.087 & -1.624 & 0.204 & -2.416 & -0.439 \\
\hline Bipower Gaussian & & & & 2.540 & -1.588 & -2.090 & -0.767 & -23.491 & -3.515 \\
\hline MedRV Gaussian & & & & & -1.730 & -2.189 & -1.049 & -11.892 & -6.148 \\
\hline Realized MN & & & & & & -0.529 & 4.604 & 0174 & $\begin{array}{l}-0.148 \\
0.510\end{array}$ \\
\hline Bipower MN & & & & & & & $\begin{array}{l}4.075 \\
4.007\end{array}$ & 0.327 & $\begin{array}{l}0.610 \\
0.688\end{array}$ \\
\hline MedRV MN & & & & & & & & -0.888 & $\begin{array}{l}0.000 \\
-0.327\end{array}$ \\
\hline Bipower Jump & & & & & & & & & 1.294 \\
\hline MedRV Jump & & & & & & & & & \\
\hline
\end{tabular}
Note: this table presents the results of the average Diebold-Mariano (1995) pairwise test statistic with 5 -. 10-. 30-. and 60-day horizon. The table reads the following way: a significant positive (negative)
estimated value rejects the null of equal performance between competing forecasts, and provides evidence in favor of model A (B). 
Table 10: Average Diebold-Mariano (1995) pairwise test statistic with 5-. 10-. 30-. and 60-day horizon for USD/JPY Futures.

\begin{tabular}{|c|c|c|c|c|c|c|c|c|c|}
\hline 5-day horizon & EGARCH & Realized Gaussian & Bipower Gaussian & MedRV Gaussian & Realized MN & Bipower MN & MedRV MN & Bipower Jump & MedRV Jump \\
\hline EGARCH & & -0.166 & -1.442 & -1.761 & -1.855 & -2.574 & -2.860 & -4.021 & -4.301 \\
\hline Realized Gaussian & & & -4.087 & -3.991 & -4.325 & -5.569 & -5.875 & -23.285 & -29.566 \\
\hline Bipower Gaussian & & & & -3.191 & -1.480 & -2.926 & -3.442 & -12.589 & -13.883 \\
\hline MedRV Gaussian & & & & & -0.952 & -2.280 & -2.795 & -11.706 & -12.657 \\
\hline Realized MN & & & & & & -4.171 & -4.638 & -10.633 & -12.495 \\
\hline Bipower MN & & & & & & & -12.403 & -6.039 & -7.003 \\
\hline MedRV MN & & & & & & & & -5.236 & -6.159 \\
\hline Bipower Jump & & & & & & & & & -4.210 \\
\hline
\end{tabular}

\begin{tabular}{|c|c|c|c|c|c|c|c|c|c|}
\hline 10-day horizon & EGARCH & Realized Gaussian & Bipower Gaussian & MedRV Gaussian & Realized MN & Bipower MN & MedRV MN & Bipower Jump & MedRV Jump \\
\hline EGARCH & & 1.592 & -1.231 & -2.051 & -4.072 & -5.276 & -5.861 & -3.823 & -4.243 \\
\hline Realized Gaussian & & & -5.097 & -4.906 & -46.915 & -81.027 & -41.709 & -20.121 & -24.834 \\
\hline Bipower Gaussian & & & & -12.436 & -1.735 & -3.403 & -3.880 & -11.716 & -13.543 \\
\hline MedRV Gaussian & & & & & -1.062 & -2.610 & -3.097 & -9.506 & -10.980 \\
\hline Realized MN & & & & & & -4.702 & -4.279 & -15.814 & -33.723 \\
\hline Bipower MN & & & & & & & -5.553 & -7.514 & -10.237 \\
\hline MedRV MN & & & & & & & & -6.541 & -9.047 \\
\hline Bipower Jump & & & & & & & & & -5.545 \\
\hline
\end{tabular}

\begin{tabular}{|c|c|c|c|c|c|c|c|c|c|}
\hline 30-day horizon & EGARCH & Realized Gaussian & Bipower Gaussian & MedRV Gaussian & Realized MN & Bipower MN & MedRV MN & Bipower Jump & MedRV Jump \\
\hline EGARCH & & 2.469 & 0.470 & -0.219 & -0.324 & -1.456 & -1.862 & -2.269 & -3.015 \\
\hline Realized Gaussian & & & -9.312 & -9.104 & -27.744 & -32.359 & -46.091 & -18.215 & -22.006 \\
\hline Bipower Gaussian & & & & -8.026 & -1.968 & -5.125 & -42.168 & -10.976 & -13.711 \\
\hline MedRV Gaussian & & & & & -0.349 & -3.260 & -44.743 & -8.542 & -10.981 \\
\hline Realized MN & & & & & & -8.782 & -7.907 & -7.388 & -10.478 \\
\hline Bipower MN & & & & & & & -4.807 & -3.345 & -5.673 \\
\hline MedRV MN & & & & & & & & -2.391 & -4.675 \\
\hline $\begin{array}{l}\text { Bipower Jump } \\
\text { MedRV Jump }\end{array}$ & & & & & & & & & -10.427 \\
\hline
\end{tabular}

\begin{tabular}{|c|c|c|c|c|c|c|c|c|c|}
\hline 60-day horizon & EGARCH & Realized Gaussian & Bipower Gaussian & MedRV Gaussian & Realized MN & Bipower MN & MedRV MN & Bipower Jump & MedRV Jump \\
\hline$\overline{\text { EGARCH }}$ & & 6.140 & $\overline{450}$ & 2.229 & & & & & \\
\hline Realized Gaussian & & & -13.920 & -13.521 & -19.590 & -24.772 & -24.024 & -25.915 & 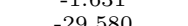 \\
\hline Bipower Gaussian & & & & -12.057 & -8.335 & -15.093 & -15.630 & -13.284 & $\begin{array}{l}-29.580 \\
-16.986\end{array}$ \\
\hline MedRV Gaussian & & & & & 1.376 & -12.496 & -12.942 & $\begin{array}{l}-8.305 \\
-8.305\end{array}$ & -16.986 \\
\hline $\begin{array}{l}\text { Realized MN } \\
\text { Red }\end{array}$ & & & & & & -13.394 & -84.304 & -8.866 & -12.164 \\
\hline Bipower MN & & & & & & & -25.172 & -2.801 & $\begin{array}{l}-13.503 \\
-6.989\end{array}$ \\
\hline MedRV MN & & & & & & & & 0.097 & $\begin{array}{l}-6.989 \\
-3.579\end{array}$ \\
\hline Bipower Jump & & & & & & & & & $\begin{array}{l}-.0 .69 \\
-13.641\end{array}$ \\
\hline MedRV Jump & & & & & & & & & \\
\hline
\end{tabular}
Note: this table presents the results of the average Diebold-Mariano (1995) pairwise test statistic with 5-. 10-. 30-. and 60-day horizon. The table reads the following way: a significant positive (negative)
estimated value rejects the null of equal performance between competing forecasts, and provides evidence in favor of model A (B). 